\section{The zebrafish eye-a paradigm for investigating human ocular genetics}

\begin{abstract}
Although human epidemiological and genetic studies are essential to elucidate the aetiology of normal and aberrant ocular development, animal models have provided us with an understanding of the pathogenesis of multiple developmental ocular malformations. Zebrafish eye development displays in depth molecular complexity and stringent spatiotemporal regulation that incorporates developmental contributions of the surface ectoderm, neuroectoderm and head mesenchyme, similar to that seen in humans. For this reason, and due to its genetic tractability, external fertilisation, and early optical clarity, the zebrafish has become an invaluable vertebrate system to investigate human ocular development and disease. Recently, zebrafish have been at the leading edge of preclinical therapy development, with their amenability to genetic manipulation facilitating the generation of robust ocular disease models required for large-scale genetic and drug screening programmes. This review presents an overview of human and zebrafish ocular development, genetic methodologies employed for zebrafish mutagenesis, relevant models of ocular disease, and finally therapeutic approaches, which may have translational leads in the future.
\end{abstract}

Eye (2017) 31, 68-86; doi:10.1038/ eye.2016.198;

published online 9 September 2016

M Moosajee, Department of Ocular Biology and Therapeutics, UCL Institute of Ophthalmology, 11-43

Bath Street, London EC1V 9EL, UK

Tel: +44 (0)20 7608 6971; Fax: +44 (0)2076086830. E-mail:m.moosajee@ucl.ac. uk

Received: 8 April 2016 Accepted in revised form: 17 June 2016

Published online:

9 September 2016
R Richardson ${ }^{1}$, D Tracey-White ${ }^{1}$, A Webster ${ }^{1,2}$ and M Moosajee ${ }^{1,2}$

large clutches of fertilised eggs ( 100-200) at weekly intervals. Fertilisation is ex utero and the developing embryo is transparent facilitating easy visualisation of early organogenesis and amenability to embryological manipulation.

Seventy per cent of human genes have at least one zebrafish orthologue, with $84 \%$ of known human disease-causing genes having a zebrafish counterpart. ${ }^{1}$ In fact, zebrafish frequently have two orthologues of mammalian genes which map in duplicated chromosomal segments as a consequence of an additional round of wholegenome duplication. The most likely fate of a duplicate gene is loss-of-function, although both copies can be retained and subfunctionalisation or neofunctionalisation can occur. Despite genome duplication, zebrafish have a similar number of chromosomes to humans (25 and 23, respectively), many of which are mosaically orthologous. These factors, in addition to the genetic versatility of the zebrafish, make it a prominent model organism for systematic mutational approaches in the study of human disease.

Advantages of the zebrafish model pertaining to the eye

The eyes of the zebrafish are large relative to the overall size of the fish, making eye bud manipulation feasible during early embryogenesis. Zebrafish are visually responsive by $72 \mathrm{~h}$ post fertilisation (h.p.f.) by which time the retina resembles adult retinal morphology that is anatomically and functionally similar to humans (Figure 1). The zebrafish retinal architecture possesses photoreceptor subtypes spatially arranged in a highly organised heterotypical photoreceptor mosaic, and, due to the diurnal nature of zebrafish, it is cone-rich akin to the human macula resulting in colour vision with a cone density close to humans. 

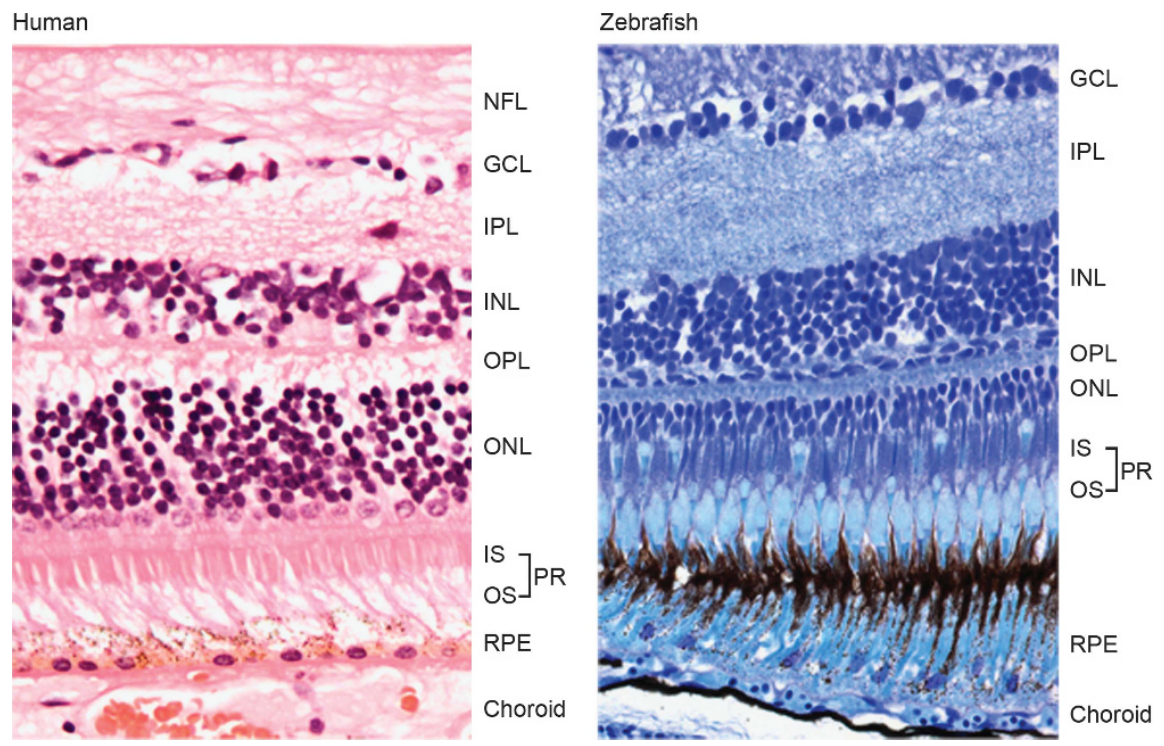

Figure 1 Cross-sectional histology of the human and zebrafish retina demonstrating similarities in the arrangement of cells and structural features that define the distinct retinal layers. RPE, pigmented epithelium; IS, inner segment; OS, outer segment; PR, photoreceptor; ONL, outer nuclear layer; OPL, outer plexiform layer; INL, inner nuclear layer; GCL, ganglion cell layer; and NFL, nerve fibre layer.

Zebrafish behaviour is an invaluable tool for assaying visual function. Zebrafish alter their skin pigmentation when exposed to different light-intensities by expanding or contracting melanosomes; if a fish has impaired vision, it perceives itself to be in an environment with low light intensity, therefore appearing hyper-pigmented. More specific visual assays take advantage of visual reflexes such as the optokinetic or startle response, and an ability to monitor visual response when varying examination conditions.

\section{Zebrafish eye morphogenesis}

Ocular development in zebrafish closely resembles that of humans and other vertebrates (Figure 2). ${ }^{2,3}$ Both develop from three distinct embryological tissues, neuroectoderm which gives rise to the neural retina, retinal pigment epithelium, optic stalk, iris dilator and sphincter muscles, and ciliary body; surface ectoderm, which forms the lens and subsequently the conjunctival and corneal epithelia; and mesenchyme which originates from the neural crest cells forming the corneal endothelium and stroma, iris stroma, ciliary muscles and vasculature, and sclera.

The formation of optic sulci as small grooves on either side of the developing forebrain by day 22 of gestation marks the establishment of rudimentary ocular development in human embryogenesis. ${ }^{4}$ During the following week of gestation, evagination of the optic sulci leads to formation of optic pits, which deepen to form optic vesicles. ${ }^{4}$ A key difference in zebrafish development is that the neural tube develops as a solid mass of cells referred to as the neural keel. ${ }^{2}$ Consequently, optic primordia evaginate from the neural keel as a dense neuroepithelial cellular mass. The optic lumina form from cavitation within the optic primordia, and these spaces expand to become continuous with the ventricles of the neural keel by 14 h.p.f. (12 somite stage). Similar to human ocular development, the zebrafish optic vesicle then undergoes a series of morphogenetic movements between 16-20 h.p.f. giving rise to a two-layered optic cup composed of retinal neuroepithelium and pigmented epithelium (Figures 2a and e). ${ }^{2,3}$

Although during both human and zebrafish development the lens placode is induced to form from the surface ectoderm cells overlying the optic cups, the morphogenetic processes resulting in placode formation differ. Cell fate tracking during zebrafish lens development suggested that delamination of the lens placode from the surface ectoderm results in formation of a solid lens mass which detaches by apoptosis of the intervening cells by 28 h.p.f. (Figures $2 \mathrm{f}-\mathrm{h}$ )..$^{5}$ In contrast, after thickening of the cells of the surface ectoderm overlying the optic vesicle at 27 days gestation, the human lens placode invaginates as a result of cessation of cell division at its centre, forming lens pits. The lens pit pinches off from the surface ectoderm as the cells of the pit delaminate from the head ectoderm and develop cellcell interactions with the opposite pit edge (Figures $2 b-d$ ).

During the fifth week of human gestation, the surface ectoderm, once separated from the lens vesicle, 
a

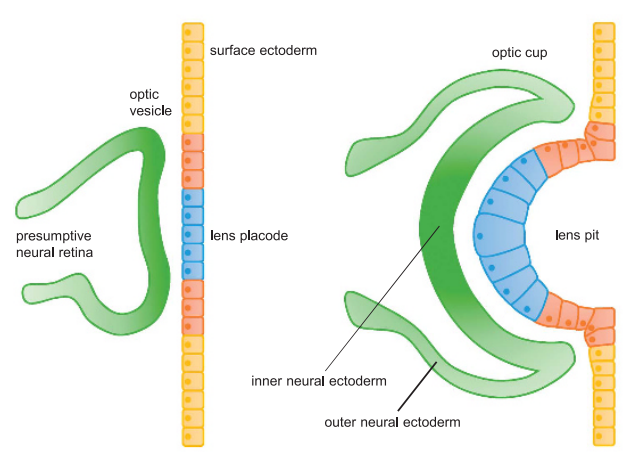

$27 d$

e

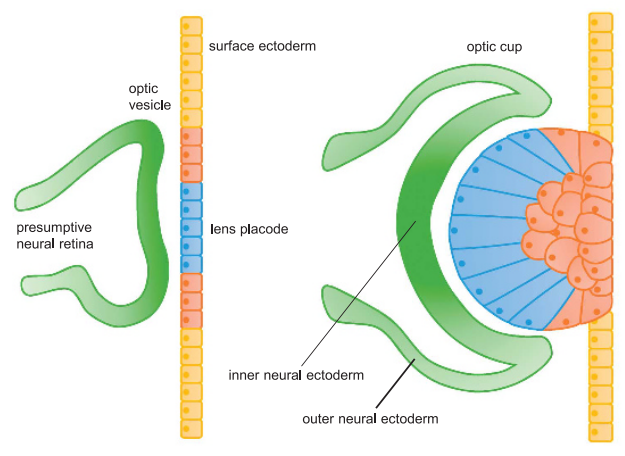

C

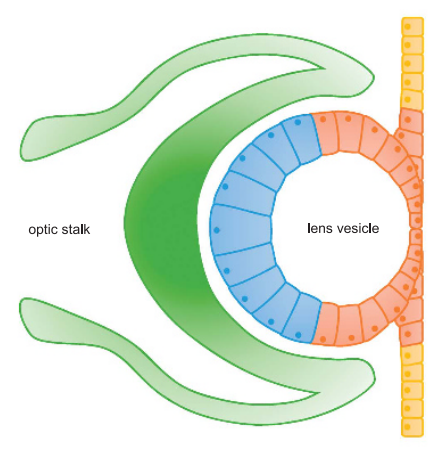

$37 d$

g

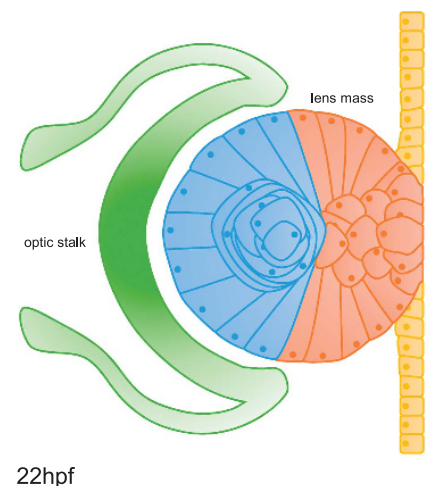

d

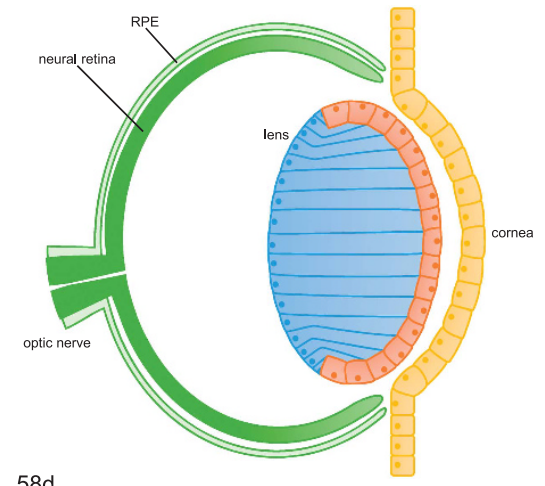

$58 d$

h

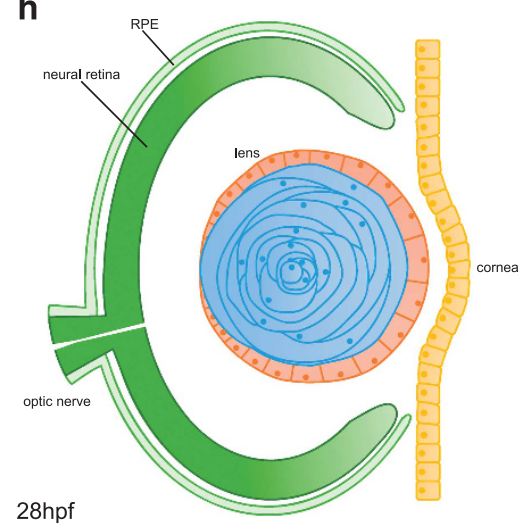

Figure 2 Schematic of human and zebrafish ocular development. The lens placode and optic vesicle are formed as the central eye field splits at 27 days of gestation in the human and 16 h.p.f. in the zebrafish (a, e). The distal portion of the optic vesicle invaginates so that the presumptive neural retina is apposed to the presumptive RPE in a double-walled cup structure (b, f). The optic cup grows circumferentially. The inner layer differentiates into the neural retina from $28-35$ days of gestation in the human and from 16 h.p.f. in the zebrafish. The outer layer of the optic cup gives rise to the RPE. The lens develops concomitantly with the retina in both human and zebrafish development. The human lens placode invaginates to become the lens pit, which deepens and closes before pinching off from the surrounding surface ectoderm (yellow) as the lens vesicle by 35 days of gestation (c). Cells of the central lens placode migrate to the posterior lens vesicle and elongate to form primary lens fibre cells (blue), filling the lens vesicle lumen by 50 days of gestation (d). Cells of the peripheral lens placode migrate to the anterior lens vesicle forming the anterior epithelium (orange). Similar to humans, the zebrafish lens begins as a lens placode (e). Progressive delamination of cells of the lens placode results in the formation of a solid lens mass by 22 h.p.f. (f, g). Cells of the central lens placode migrate to the posterior lens mass, elongate, and differentiate to form primary lens fibre cells (blue). Cells of the peripheral lens placode migrate to the anterior lens mass to form the anterior epithelium (orange). In both human and zebrafish ocular development, the cornea (yellow) develops when the surface ectoderm closes after detachment of the lens vesicle or lens mass, respectively, from the surface ectoderm $(\mathrm{d}, \mathrm{h})$. Human ocular development is represented here based on the widely accepted mammalian lens cell fate map.

differentiates into the corneal epithelium. ${ }^{4}$ This process occurs by 30 h.p.f. during zebrafish embryogenesis. ${ }^{6}$ Simultaneously, the corneal endothelial monolayer forms as migratory periocular mesenchymal cells migrate into the cornea from peripheral regions of the optic cup between 30-36 h.p.f. ${ }^{6}$ Subsequently, the neuroectodermal layers of the optic vesicle invaginate ventrally, meeting across the optic fissure along the proximo-distal axis, encircling the optic stalk by the end of the 4 th week of human gestation and by 24 h.p.f. in the zebrafish. The retina and retinal pigment epithelium are confined within the optic cup. Closure of the optic fissure is achieved by the seventh week of gestation during human embryogenesis and by 48 h.p.f. in the zebrafish. By these time points morphogenesis of the eye is largely complete with only retinal neurogenesis proceeding.

\section{Vasculature}

Similar to transient hyaloid vascular anatomy in mammalian embryos, the primitive zebrafish retinal vasculature branches from the central retinal artery by angiogenesis between 24-29 h.p.f. The optic artery enters the eye ventrally through the optic fissure and forms the 
single loop of the hyaloid artery within the eye, which exits the optic fissure as the hyaloid vein. Endothelial cells between the lens and retina give rise to the first hyaloid vessel which is distinguishable as rudimentary vasculature by 60 h.p.f. The hyaloid vessels branch and lose contact with the lens, adhering to the inner limiting membrane of the juvenile retina by 30 d.p.f. In mammals, remodelling of the hyaloid vessels involves a coordinated process of hyaloid regression and retinal angiogenesis. In zebrafish, the vessels gradually move away from the lens and deflect onto the retina as the vitreous forms; remodelling of the vessels is sufficient to establish retinal vasculature in the zebrafish retina without re-growth, perhaps due to the close proximity of the lens to the retina.

\section{Retinal neurogenesis}

Human retinal neurogenesis is initiated during gestational week six. The ganglion cells exit the cell cycle and differentiate to establish the innermost layer of the retina by 20 weeks gestation. ${ }^{4}$ Similarly, the ganglion cells are the first identifiable cells in the developing zebrafish retina. Differentiation of ganglion cells is initiated in the ventronasal retina and spreads dorsally to the ventrotemporal retina. ${ }^{2,7}$ The first axons from the ganglion cells exit the retina and by 40 h.p.f. reach the optic tectum. Ganglion cell differentiation is closely followed by the appearance of amacrine and horizontal cells. Lamination of the retina progresses rapidly, spreading across most of the retina by 48 h.p.f. ${ }^{3}$ During the 10th week of gestation, photoreceptor cell bodies become apparent in the outer nuclear layer of the human retina, developing radially from the optic stalk. In the zebrafish retina, opsin expression can be detected in the ventral patch by 50 h.p.f., closely followed by the appearance of rod and cone outer segments and synaptic terminals by 55 h.p.f. ${ }^{3}$ Rod cells and Müller glial cells are the last to differentiate in both human and zebrafish retinal development. During human embryogenesis, visual evoked potentials can be recorded by 30 weeks gestation suggestive of a level of functional integrity. The zebrafish exhibits visual function by 72 h.p.f, with most major classes of cell being identifiable in the central retina..$^{3,7,8}$ Interestingly, by $72-74$ h.p.f a specialised area in the temporal retina develops which is characterised by a high density of cones and corresponding reduction in rods. This 'area temporalis' is similar to the fovea centralis in humans, but has been suggested to provide zebrafish with an area of better visual acuity in their anterior visual field. ${ }^{3}$ A visual evoked startle response (body twitch induced by an abrupt decrease in light intensity) and electroretinogram can be generated by 72 h.p.f. ${ }^{8}$

\section{Mature retinal anatomy}

Comparable to the human eye, the mature zebrafish retina is composed of three nuclear layers separated by two plexiform layers. The photoreceptor rod and cone cell bodies reside in the outer nuclear layer; the amacrine, horizontal, and Müller glial cell bodies occupy the inner nuclear layer and the ganglion cell bodies are contained in the ganglion cell layer. Synapsis between these nuclear layers occurs at the plexiform layers. Larval zebrafish vision is mediated almost entirely by cone photoreceptors; zebrafish possess blue, UV-sensitive, and red-green double cones and one rod cell type anatomically arranged in a mosaic pattern. ${ }^{9}$ Short cones become identifiable by 4 d.p.f. and by 12 d.p.f., all photoreceptor types are discernible based on the morphological criteria. In contrast, the human retina lacks UV-sensitive cones.

\section{Gene discovery}

The usefulness of the zebrafish model in ocular research originates not only from the striking anatomical similarities in ocular development between human and zebrafish embryos, but also from its amenability to experimental and genetic manipulation. Initial large-scale forward genetic screens in the 1990's involved mutagenesis with ethylnitrosourea and facilitated the isolation of over 2000 mutations affecting the first 5 days of zebrafish development. Included in the spectrum of mutant phenotypes obtained were multiple ocular defects. ${ }^{10}$ More recently, targeted genetic screens have been employed to isolate mutations specifically affecting eye development. Such screening methods have involved behaviour assays, examination of eye morphology or the use of transgenic reporters.

\section{Zebrafish mutagenesis}

The development of sophisticated mutagenesis techniques, for example morpholino anti-sense oligonucleotide knockdown, transcription activator-like effector nucleases (TALENs) or the clustered regularly interspaced short palindromic repeats (CRISPR) system, has facilitated the identification of multiple zebrafish mutants that model human genetic eye disease.

Zebrafish transgenesis can be achieved simply by the injection of purified plasmid DNA into newly fertilised eggs, however, this approach does have drawbacks. The potential to induce mosaic distribution of injected DNA into injected embryos, and late transgene integration at high copy numbers or inefficient incorporation of the transgene into genomic DNA can make generation of transgenic zebrafish lines a laborious 
task. The development of autonomous and nonautonomous transposable elements, has improved the efficiency of germline transformation. ${ }^{11}$

Injection of an anti-sense oligonucleotide morpholino which is complementary to a specific mRNA of interest into fertilised zebrafish eggs, can inhibit its expression by sterically blocking the translation initiation complex to hinder ribosome assembly or by binding and blocking sites involved in pre-mRNA processing via inhibition of the splicesome components. Despite highly efficient gene knockdown in early development, the transient activity of an injected morpholino oligonucleotide rarely persists beyond 5 d.p.f. This is however, sufficient for the timeframe of zebrafish ocular development. Morpholino anti-sense oligonucleotides have been an indispensible tool in studying gene function during both anterior and posterior segment development, although the reliability and reproducibility of the results are subject to increasing scrutiny with concerns raised with regard to poor correlation between morpholino-induced and mutant phenotypes. In addition, the constitutive activity of these oligonucleotides limits their use for applications where embryonic development depends on specific temporal and spatial control of gene function; deciphering the molecular mechanisms that underpin complex developmental processes requires methods for perturbing gene expression with similar precision. Photo-cleavable morpholino oligonucleotides allow the activation or deactivation of morpholino function by UV exposure. ${ }^{12}$ Recently, RNA-interference mediated chromatin silencing has been used to induce sequence-specific gene knockdown by convergent transcription (in which a DNA sequence is simultaneously transcribed in sense and anti-sense orientations directed by two inducible promoters) in zebrafish. ${ }^{13}$

The targeted introduction of mutations using sequencespecific TALENs or the CRISPR system have recently been successfully applied to generate loss-of-function alleles by specifically targeting open reading frames or deletion or inversion of whole-chromosomal regions in vivo with efficiencies in zebrafish similar to those obtained using zinc-finger nucleases and transcriptionlike nucleases. ${ }^{14}$

TALENs comprise a non-specific DNA-cleaving nuclease fused to an engineered DNA-binding domain, which contains a series of tandem repeats. Binding of two TALENs to their respective target sites, reconstitutes the active nuclease domain resulting in cleavage of the targeted genomic locus by inducing a double-strand break. Subsequent DNA repair by homologous recombination or non-homologous end joining mediates DNA insertion, deletion, and replacement, or introduces frame-shift mutations respectively. In the zebrafish, TALENs has been used to induce somatic tissue mutations in the golden ( $\mathrm{gol}$ ) gene, which encodes a putative cation exchanger (slc24a5) required for pigmentation in the embryo. Intense melanosome production occurs in the zebrafish RPE at 2 d.p.f., however, homozygous null gol mutants appear pigmentless at this stage providing a robust method to assess somatic loss of gene function. Almost all TALEN RNA-injected wild-type embryos displayed mutant non-pigmented cells in the RPE, highlighting the efficiency with which TALENs can induce directed mutations in the zebrafish. ${ }^{15}$

CRISPR-Cas is a microbial adaptive immune system that uses targeted nucleases to initiate double-strand breaks in foreign genetic elements. CRISPR RNAs that guide the Cas9-ribonucleoprotein complex to the target sequence can be injected into the zebrafish embryo and designed to target any 20 nucleotide genomic sequence to achieve phage or plasmid DNA cleavage with high specificity. The potential of CRISPR-mediated gene targeting has recently been demonstrated in a large-scale reverse genetic screening strategy to identify zebrafish genes involved in electrical synaptogenesis. ${ }^{16}$ Multiplexed pool guide RNA injections were designed to simultaneously examine multiple loci, allowing the identification of phenotypes induced by several gene deletions. With the breadth of accessible ocular phenotypes in zebrafish, such strategies could prove invaluable in the identification of candidate eye disease genes. This is particularly important in gene-knockdown investigations where the anticipated phenotype arises after the 5 d.p.f. efficacy window of morpholinos.

\section{Zebrafish models of human ocular disorders}

\section{Ocular coloboma}

Failure of optic fissure closure underpins the aetiology of ocular coloboma, which is characterised by the persistence of a cleft potentially spanning the iris, ciliary body, zonules, retina, choroid, and optic nerve. Ocular coloboma has been reported in up to $11.2 \%$ of blind children worldwide, with an estimated incidence of between $0.5-7.5$ per 10000 births and is often associated with other congenital anomalies of the eye. ${ }^{17}$ Mutations in PAX2, CHD7, SOX2, PAX6, GDF6, YAP1 OTX2, SHH, SIX3, FADD, MAF, ZFHX1B, RX, GDF3, MAB21L2, $S A L L 2$, and $A B C B 6$, as well as multiple chromosomal aberrations have been associated with coloboma, although the molecular mechanisms that underpin this condition remain to be elucidated.

Zebrafish models of ocular coloboma have provided a vital paradigm for understanding optic fissure morphogenesis, with mutations in zebrafish orthologues of human ocular coloboma-causative genes resulting in an 
Table 1 Summary of existing zebrafish models of human ocular disorders for which the genetic basis is known and associated human homolog

\begin{tabular}{|c|c|c|c|}
\hline Ocular phenotype & Zebrafish mutant/morphant & Affected zebrafish gene (gene symbol) & $\begin{array}{l}\text { Human } \\
\text { homologue }\end{array}$ \\
\hline \multirow[t]{13}{*}{ Coloboma } & apc & adenomatous polyposis coli (apc) & $\mathrm{APC}$ \\
\hline & blowout (blw) & patched1 (ptch1) & PTCH1 \\
\hline & lamb1a, grumpy & laminin, $\beta$ 1a (lamb1a) & LAMB1 \\
\hline & lamc1, sleepy & laminin, $\gamma 1$ (lamc1) & LAMC1 \\
\hline & cdh2, glass onion (glo) & cadherin 2 , type $1, \mathrm{~N}$-cadherin (neuronal) (cdh2) & $\mathrm{CDH} 2$ \\
\hline & tfap2a & transcription factor ap2 alpha & TFAP2A \\
\hline & No isthmus (noi) & paired box gene 2a (pax2a) & PAX2 \\
\hline & bcor & bcl6 co-repressor (bcor) & BCOR \\
\hline & gdf6a & growth differentiation factor $6 a$ (gdf6a) & GDF6 \\
\hline & zic2a & zic family member 2a (zic2a) & ZIC2 \\
\hline & znf703 & zinc-finger protein 703 (znf703) & ZNF703 \\
\hline & znf503 & zinc-finger protein 503 (znf503) & ZNF503 \\
\hline & fadd & Fas-associated via death domain (fadd) & FADD \\
\hline \multirow{6}{*}{$\begin{array}{l}\text { Microphthalmia/ } \\
\text { anophthalmia }\end{array}$} & gdf6as327/s327, dark half & gdf6a & GDF6 \\
\hline & gdf6am $233 / \mathrm{m} 233$, out of sight (out) & gdf6a & GDF6 \\
\hline & $\mathrm{rx}$ & retinal homeobox gene (rx) & RAX \\
\hline & $\operatorname{six} 6 b$ & SIX homeobox 6b (six6b) & SIX6 \\
\hline & pax6b, sunrise (sri) & paired box 6b (pax6b) & PAX6 \\
\hline & pax6a & paired box 6a (pax6a) & PAX6 \\
\hline \multirow[t]{3}{*}{ Cyclopia } & ndr2, cyclops (сус) & nodal-related 2 (ndr2) & NODAL \\
\hline & ndr1, squint (sqt) & nodal-related 1 (ndr1) & NODAL \\
\hline & one-eyed pinhead (oep) & teratocarcinoma-derived growth factor 1 (tdgf1) & TDGF1 \\
\hline \multirow{9}{*}{$\begin{array}{l}\text { Corneal dystrophies/ } \\
\text { cataract }\end{array}$} & lama1, bashful & laminin, $\alpha 1$ (lama1) & LAMA1 \\
\hline & lamb1a, grumpy & laminin, $\beta$ 1a (lamb1a) & LAMB1 \\
\hline & lamc1, sleepy & laminin, $\gamma 1$ (lamc1) & LAMC1 \\
\hline & cdipt, lens opaque (lop) & $\begin{array}{l}\text { CDP-diacylglycerol-inositol 3-phosphatidyltransferase } \\
\text { (cdipt) }\end{array}$ & CDIPT \\
\hline & $\operatorname{cdh} 4$ & cadherin 4 , type 1 , R-cadherin (retinal) (cdh4) & $\mathrm{CDH} 4$ \\
\hline & ints7 & integrator complex subunit 7 (ints7) & INTS7 \\
\hline & $\mathrm{rbm} 42$ & RNA binding motif protein 42 (rbm42) & RBM42 \\
\hline & uhfr1 & $\begin{array}{l}\text { Ubiquitin-like, containing PHD and ring finger } \\
\text { domains, } 1 \text { (uhrf1) }\end{array}$ & UHRF1 \\
\hline & wdr36 & WD repeat domain 36 (wdr36) & WDR36 \\
\hline \multirow[t]{5}{*}{ Vasculature } & plxnd1, out of bounds (obd) & plexin D1 (plxnd1) & PLXND1 \\
\hline & lama1, bashful & laminin, $\alpha 1$ (lama1) & LAMA1 \\
\hline & vps11, platinum (plt) & vacuolar protein sorting 11 (vps11) & VPS11 \\
\hline & vhl & von Hippel-Lindau tumour suppressor (vhl) & VHL \\
\hline & lama1, bashful & laminin, $\alpha 1$ (lama1) & LAMA1 \\
\hline \multirow[t]{16}{*}{ Retinal and RPE disease } & $\mathrm{chm}^{\mathrm{ru} 848 / \mathrm{ru} 848}$ & choroideremia (chm) & $\mathrm{CHM}$ \\
\hline & prkci, heart and soul & protein kinase $\mathrm{C}$, iota (prkci) & PRKCl \\
\hline & fading vision ( $\mathrm{fdv}$ ) & premelanosome protein a (pmela) & PMEL \\
\hline & vps39, leberknödel (lbk) & vacuolar protein sorting 39 homologue (vps39) & VPS39 \\
\hline & gucy2f & guanylate cyclase $2 \mathrm{~F}$, retinal (gucy2f) & GUCY2D \\
\hline & crb2a, oko meduzy (ome) & crumbs family member 2a (crb2a) & CRB2 \\
\hline & ift88, oval (ovl) & intraflagellar transport 88 homologue (ift88) & IFT88 \\
\hline & myo7aa, mariner (mar) & myosin VIIAa (myo7aa) & MYO7A \\
\hline & ush1 $\mathrm{c}^{\text {fh2 } 298}$ & usher syndrome 1C (ush1c) & USH1C \\
\hline & sputnik & cadherin-related 23 (cdh23) & $\mathrm{CDH} 23$ \\
\hline & orbiter & protocadherin-related $15 a(p c d h 15 a)$ & PCDH15 \\
\hline & dctn1a, mikre oko & dynactin 1a (dctn1a) & DCTN1 \\
\hline & dctn2, ale oko & dynactin 2 (dctn2) & DCTN2 \\
\hline & ift88, oval (ovl) & intraflagellar transport 88 homologue (ift88) & IFT88 \\
\hline & mpp5a, nagie oko & membrane protein, palmitoylated $5 a(m p p 5 a)$ & MPP5 \\
\hline & pde6c, eclipse & phosphodiesterase 6C, cGMP-specific, cone, alpha, & PDE6C \\
\hline
\end{tabular}


Table 1. (Continued)

\begin{tabular}{llll}
\hline Ocular phenotype & Zebrafish mutant/morphant & Affected zebrafish gene (gene symbol) & $\begin{array}{l}\text { Human } \\
\text { homologue }\end{array}$ \\
\hline & traf3ip1, elipsa & $\begin{array}{l}\text { TNF receptor-associated factor 3 interacting protein 1 } \\
\text { (traf3ip1) }\end{array}$ & TRAF3IP1 \\
& epb4115, mosaic eyes (moe) & $\begin{array}{l}\text { erythrocyte membrane protein band 4.1 like 5 } \\
\text { (epb4115) }\end{array}$ & EPB41L5 \\
& tyrosinase (tyr) & TYR \\
tyr, sandy (sdy) & solute carrier family 45 member 2 (slc45a2) & SLC45A2 \\
slc45a2, albino (alb) & solute carrier family 24 member 5 (slc45a5) & SLC24A5 \\
slc24a5, golden (gol) & chromosome 10 open reading frame 11 (c10orf11) & C10ORF11 \\
c10orf11 & retinitis pigmentosa 2 (rp2) & RP2 \\
rp2 & retinitis pigmentosa GTPase regulator (rpgr) & RPGR \\
rpgr & ceramide kinase-like (cerkl) & CERKL \\
cerk1 & chromosome 2 open reading frame 71 (c2orf71) & C2ORF71 \\
c2orf71 & &
\end{tabular}

array of observed retinal and lens defects (Table 1). PAX2 mutations are one of the most commonly identified genetic causes of renal-coloboma syndrome. ${ }^{18}$ Zebrafish mutants which are homozygous for pax $2 a$ mutations exhibit optic fissure closure defects and lack the midbrain, midbrain-hindbrain boundary, and cerebellum, fail to feed and die within 2 weeks. ${ }^{19}$ This optic fissure closure defect has been attributed in part, to the inhibition of downstream effector caspases and deficiencies in the control of cellular proliferation, implicating a role for pax $2 a$ in the fine tuning of apoptotic cell death. ${ }^{20,21}$

Recessive mutations in several other genes have been associated with ocular coloboma including laminin- $\beta 1$ (lamb1a), laminin- $\gamma 1$ (lamc1), n-cadherin (ncad), adenomatous polyposis coli $(a p c)$, growth and differentiation factor 6 ( $g d f 6 a)$ and patched1 (ptc1). ${ }^{22-24}$ Morphological defects exhibited by lamb1 and lamc1 mutants can likely be attributed to pathological changes in extracellular matrix deposition or cell-ECM interactions, where laminin proteins are a major component of the ocular basal lamina, having a critical role in determining and maintaining tissue function. ${ }^{25}$ In addition, defective regulation of retinal progenitor cell number and proliferative activity and abnormal activation of apoptotic cell death pathways may contribute to persistence of the optic fissure in these mutants. ${ }^{20,23}$

\section{Microphthalmialanophthalmia}

Microphthalmia is characterised by an eye with total axial length that is at least two standard deviations below the mean for age, and anophthalmia refers to complete absence of ocular tissue in the orbit. Although the aetiology of microphthalmia/anophthalmia is complex, heterozygous loss-of-function mutations in SOX2 or OTX2 have been described as the most prevalent monogenic cause to date. ${ }^{26}$ Microphthalmia-causing mutations have also been described in CHX10, OTX2, PAX6, RAX, BMP4, GDF6, and GDF3.

During zebrafish ocular development $g d f 6$ expression is limited to the dorso-temporal retina, and morpholinoinduced knockdown results in variable retinal defects including decrease in lenticular and ocular size, loss of retinal lamination, and vacuolation of the lens. ${ }^{27}$ The small eye phenotype in the dark half ${ }^{327}$ mutant has been attributed to a nonsense mutation in the $g d f 6 a$ gene which causes retinotectal mapping defects. ${ }^{28}$ Research suggests that a gradient of gdf6a signalling works in conjunction with Sonic Hedgehog (Shh) to establish dorsal-ventral positional information in the retina. Similarly, a mutation in the zebrafish out of sight (out $)^{m 233}$ locus, which eliminates the initiation codon in $g d f 6 a$, results in a severe small eye phenotype, likely caused by a transient wave of apoptosis at the onset of neurogenesis. ${ }^{29}$

Morpholino-induced knockdown of $r \times 1$ and $r \times 2$ in zebrafish results in optic vesical formation, but microphthalmia ensues. ${ }^{30}$ Conversely, knockdown of the zebrafish paralogue $r x 3$ results in an eyeless phenotype and associated expanded forebrain suggesting a role for $\mathrm{Rx} 3$ in the regulation of spatiotemporal expression of eye field transcription factors during optic vesicle morphogenesis. ${ }^{31}$

PAX6 haploinsufficiency in humans is the main cause of aniridia, but in rare cases results in microphthalmia. In zebrafish, two pax6 paralogues, pax6a and pax6b, encode functionally redundant proteins involved in formation and differentiation of the retina and lens. Zebrafish homozygous for the pax6b missense mutation sunrise (sri), display a mild microphthalmia phenotype which manifests in anterior segment dysgenesis. ${ }^{32}$ Interestingly, morpholino-induced knockdown of pax $6 a$ or simultaneous knockdown of pax $6 a$ and pax $6 b$ results in a similar but more severe phenotype; morphants present with reduced body size and neural tube girth, 
morphologically abnormal brain development, and microphthalmia. ${ }^{33}$

TALENs-mediated knockdown of pax $6 a$ and pax $6 b$ simultaneously, by targeting conserved sequences between these paralogs, resulted in eye phenotypes including microphthalmia, closely recapitulating the ocular morphant phenotype described. ${ }^{34}$ The strategy employed not only confirms the efficiency of TALENsmediated gene disruption, but also emphasises the ability to induce bi-allelic and paralogous genome editing, which is important when investigating genes that are duplicated in the zebrafish genome.

\section{Cyclopia}

Cyclopia is characterised by the presence of a single eye with variable degrees of doubling of the intrinsic ocular structures. During embryogenesis, the splitting of the eye field occurs in parallel with establishment of the midline, a process mediated by $\mathrm{SHH}$. Mutations in $\mathrm{SHH}$ cause midline patterning defects, which manifest as holoprosencephaly and cyclopia.

Zebrafish express three mammalian $\mathrm{SHH}$ orthologues in the embryonic midline, sonic you (syu), tiggy-winkle hedgehog (twhh), and echidna hedgehog (ehh). Zebrafish with simultaneous morpholino-induced knockdown of syu and twhh function, exhibit partial cyclopia suggesting that zebrafish contain two functionally redundant orthologues of mammalian $S H H$, with similar roles in anterior midline patterning. ${ }^{35}$

Additional zebrafish cyclopic mutations have been described. The zebrafish loci squint (sqt) and cyclops $(c y c)$ encode Nodal-related proteins, Ndr1 and Ndr2, respectively. Both squint and cyclops mutations lead to severe defects in the development of the medial floor plate and ventral brain; mutants lack head and trunk mesoderm, and endoderm derivatives which leads to cyclopia as a consequence of incomplete splitting of the eye field. ${ }^{36}$ Similarly, the zebrafish one-eyed pinhead (oep) mutation also results in cyclopia and defects in the formation of endoderm, prechordal plate, and ventral neuroectoderm. ${ }^{37}$ Interestingly, the oep gene encodes an epidermal growth factor-related protein which acts as a co-receptor in the Nodal pathway, ${ }^{37}$ hence, linking the three zebrafish cyclopic mutations $c y c$, sqt, and oep in a common signalling pathway central to mesendoderm formation and development. Importantly, research suggests that Nodal signalling acts upstream of the Shh pathway during establishment of zebrafish ventral forebrain patterning indicating that known causative zebrafish cyclopic genes converge in a final common pathway.

Importantly, a novel strategy for heritable chromosomal editing in the zebrafish has recently been described using nodal-related genes; TALENs was used simultaneously with ZFNs to induce whole locus and transcription start site element-specific deletions in the zebrafish sqt and cyc genes. ${ }^{38}$ Cyclopia and midline defects were observed in both sqt and cyc nucleaseinjected embryos, consistent with previously reported mutant embryos, demonstrating the ability to induce specific deletions at desired locations with higher efficacy using the combined action of differing nuclease pairs.

\section{Glaucoma}

Glaucoma is an optic neuropathy that can result in progressive and irreversible vision loss secondary to retinal ganglion cell death and subsequent optic nerve head damage. Many human genes have been implicated in glaucoma, although the incomplete penetrance reported within families is indicative of a multifactorial aetiology. Despite dissimilarity between the mammalian trabecular meshwork and the zebrafish annular ligament ${ }^{39}$ and the vectorial (flowing from dorsal to ventral) flow of zebrafish aqueous humour which contrasts the circumferential flow of aqueous humour around and through the iridocorneal angle in mammals, the anatomy and overall ultrastructure of the tissues and cells facilitating aqueous humour dynamics in zebrafish, show conservation with that of mammals. ${ }^{40}$

Adult zebrafish and mammals share similar intraocular pressure (IOP) distributions. ${ }^{41}$ With this, the disease-risk phenotype of glaucoma has been well studied in zebrafish; the reduced melanin zebrafish mutant brass (genetic basis unknown) exhibits mildly elevated IOP and iris hypoplasia whilst the bugeye mutant, which has a recessive mutation in the gene encoding low-density lipoprotein receptor related protein 2 (Lrp2), shows chronic elevated IOP resulting in enlarged eyes, retinal stretch, and RGC loss with progressive optic nerve damage. ${ }^{41,42}$

In humans, mutation of the forkhead C1 (FOXC1) transcription factor gene results in a spectrum of anterior segment dysgenesis phenotypes, including glaucoma in some individuals. ${ }^{43}$ Zebrafish foxc1 expression is limited to the anterior segment and periocular mesenchyme, ${ }^{44}$ and has been implicated in the regulation of factors that mediate responses to oxidative stress and suppression of apoptosis in cells involved in aqueous humour dynamics. ${ }^{45}$ In addition, loss of foxC1 results in defects of the vascular basement membrane integrity indicating that genetic interactions between fox $\mathrm{C} 1$ and basement membrane components influence vascular stability which may impact glaucoma pathogenesis. ${ }^{46}$

Zebrafish were employed as a model to determine the normal nucleolar function of wdr36, a gene previously identified as causative for human primary open-angle 
glaucoma (POAG). ${ }^{47}$ From this work, a role for wdr36 in ribosomal RNA processing was established and subsequently confirmed in other species, highlighting the importance of zebrafish in the rapid functional elucidation of glaucoma-associated proteins. Similarly, mutations in the human LIM-homeodomain gene LMX1B are associated with Nail-Patella syndrome and an increased susceptibility to POAG. ${ }^{48}$ Zebrafish $\operatorname{lm} x 1 b .1$ and $\operatorname{lm} x 1 b .2$, orthologues of the mammalian $L M X 1 B$ gene, are expressed in cells of the periocular mesenchyme and anti-sense morpholinos against $\operatorname{lm} x 1 b .1$ and $\operatorname{lm} x 1 b .2$ not only result in defective migration of periocular mesenchymal cells and subsequent apoptosis, but also alter the expression of foxc $1 .{ }^{49}$

\section{Corneal dystrophies}

Human genetic corneal dystrophies, although uncommon, can cause significant visual impairment when corneal transparency is lost. At 6 m.p.f., the zebrafish cornea contains all five major layers found in the human cornea. ${ }^{6}$ A number of genes associated with human corneal dystrophies encode proteins which are expressed in the zebrafish cornea including tgfb1, keratin 3 , and corneal keratan sulfate proteoglycan. ${ }^{50}$ Importantly, some human corneal dystrophy-linked genes show conserved expression in the zebrafish cornea, including the François-Neetens fleck corneal dystrophygene PIP5K3. 51

The human gene Keratocan (KERA) encodes a corneal small leucine-rich proteoglycan which is essential in maintaining corneal transparency. Mutations in KERA are directly associated with inherited cornea plana, which manifests clinically as reduced visual acuity due to a flattened convex corneal curvature and corneal parenchymal opacity. ${ }^{52}$ The zebrafish homologue zKera is widely expressed in the brain and ocular tissue at larval stages and limited to the cornea in the adult suggestive of an important role in the maintenance of corneal transparency and structure. ${ }^{53}$

The surface of the human eye is covered by corneal epithelial cells, which regenerate from a small population of limbal epithelial stem cells (LESCs) found in limbal epithelial crypts at the peripheral cornea. ${ }^{54}$ The human $L A M A 1$ gene encodes an important basal membrane protein which is highly expressed in LESCs. ${ }^{55}$ Interestingly, loss of the functional zebrafish homologue lama1 in ocular tissues leads to focal corneal dysplasia in adult zebrafish. ${ }^{56}$

\section{Cataract}

In conjunction with the cornea, the transparent lens functions as a key refractive element required to focus light on the retina. Cataract is an opacity in the lens and represents the leading cause of preventable sight loss worldwide. Although most cases of cataracts are age-related and likely have both genetic and environmental causes, congenital cataracts occur in 2-3 per 10000 live births, often presenting during the first year of life. ${ }^{57}$ The causative genes for many cases of human cataract remain unknown.

Several mutations affecting the zebrafish lens have been described. The lens opaque (lop) mutation which lies within the cdipt (phosphatidylinositol synthase) gene, causes cataract as a consequence of disrupted lens fibre differentiation and unregulated cell proliferation leading to retinal photoreceptor degeneration in the mutant. ${ }^{58}$ Large-scale zebrafish mutagenesis screens have identified many more zebrafish which present with lens defects as a consequence of mutations in genes which encode crystallins, connexions, aquaporin, beaded filament proteins, and heat-shock factors. These proteins are important in maintaining the transparency and refractive power of the lens and the cornea. The cloche zebrafish mutant (genetic basis unknown) presents with cataract as a consequence of defects in haematopoiesis and vascular development due to $\gamma$-Crystallin insolubility and defective lens fibre cell differentiation. ${ }^{59}$ Interestingly, the cataract phenotype was rescued by overexpression of $\alpha$-Crystallin, a protein that shows reduced expression in the cloche mutant lens. Importantly, mutations in lens crystallins have also been associated with cases of human cataract and current research focuses on therapeutic strategies that may revive lens transparency by pharmacological targeting to safeguard crystalline chaperone activity, thereby evading the need for cataract surgery.

The lens capsule contains a heterotrimer of $\alpha, \beta$, and $\gamma$ subunits of the extracellular matrix component laminin. Mutations in human $L A M B 2$ result in severe renal disease coupled to multiple ocular defects including lens malformation and cataract. ${ }^{60}$ Similarly, mutations in zebrafish lama1, lamb1, and lamc1 genes result in retinal lamination and lens defects including ectopic position of the lens within the retina, loss of lens capsule integrity, and lens fragmentation, highlighting the importance of laminin in lens development and structural integrity. ${ }^{25,61}$ Extracellular matrix-dependent myosin dynamics also contribute to the cytoskeletal organisation of the lens. Heterozygous mutations in the myosin chaperone UNC $45 B$ have recently been associated with congenital cataract. ${ }^{62}$ Zebrafish steif mutants carrying an $u n c 45 b$ nonsense mutation present with a small lens and lens fibre cell nuclear retention, where degradation of all cytoplasmic organelles within the lens fibre cells is necessary for the establishment and maintenance of normal lens transparency. Injection of RNA encoding the human UNC45B protein into homozygous mutant 
zebrafish embryos rescued the ectopic nuclei phenotype, highlighting the potential of novel therapeutic targets to treat ocular pathologies.

\section{Aniridia}

Aniridia is a pan-ocular disease that manifests by alterations in the structure and function of the eye, including variable degrees of iris hypoplasia, glaucoma, foveal hypoplasia, nystagmus, glaucoma, cataract, and corneal abnormalities. This congenital eye malformation has been linked to haploinsufficiency of human PAX 6 and aberrations in the genomic region downstream of $P A X 6,{ }^{63}$ with different clinicopathological phenotypes determined by PAX6-mutation dosage. A point mutation in the PAX6 regulatory cis-element (SIMO), that resides in intron 9 of the gene ELP4, positioned downstream of the PAX6 promoter, has been described in a familial case of classical aniridia. ${ }^{64}$ Interestingly, SIMO cis-element-driven reporter expression was apparent in the lens, and more variably in the diencephalon and hindbrain of a transgenic zebrafish reporter line by 48 h.p.f., suggestive of the importance of this conserved cis-regulatory element in zebrafish ocular development. ${ }^{64}$ Deletion of the SIMO element from the pax 6 zebrafish locus resulted in complete abolition of Pax6 reporter expression in the lens, despite the persistence of other controlled lens-specific enhancers, indicating that the SIMO enhancer element is specifically important for maintained tissue-specific expression from pax6 promoters during ocular development. This work supports the notion of the existence of conserved cis-regulatory mechanisms that govern gene expression in both human and zebrafish developing ocular tissues.

\section{Ocular albinism and associated syndromes}

Oculocutaneous albinism (OCA; affects the eyes, skin, and hair) and ocular albinism (affects only the eye) are recessive pigmentation disorders caused by defective melanin synthesis or trafficking which manifest in a broad phenotypic range. OCA type 1 and 3 are associated with mutations in tyrosinase (TYR) and tyrosinase-related protein 1 (TYRP1), both of which are directly involved in melanin synthesis. OCA type 2 is associated with mutations in OCA2 which encodes melanosomal transmembrane protein. OCA type 4 and 6 are caused by mutations in SLC45A2 and SLC24A5, respectively, which encode melanosomal membrane-associated transporter proteins. OCA type 7 is due to mutations in the C10ORF11 gene. Knockdown of the zebrafish orthologues tyr (TYR), oca21p (OCA2), tyrp1a and tyrp1b simultaneously (TYRP1), slc45a2 (SLC45A2), slc24a5 (SLC24A5), or c10orf11 (C10ORF11) lead to a reduction or complete absence of melanin in the eye, with varying responses to visual testing immediately after light exposure, phenocopying the varying degrees of hypopigmentation of the eyes of OCA patients and exemplifying the use of zebrafish to model human pathologies of the eye. ${ }^{65-69}$

Multiple zebrafish mutants have been identified with visual defects secondary to functional deficits in the RPE. The vps39 mutant carries a mutation in the zebrafish orthologue of VPS39, a gene encoding a component of the vacuole protein sorting (HOPS) membrane-tethering complex, which coordinates vesicle fusion and transport. ${ }^{70}$ The ops39 mutant presents with hypopigmentation of skin melanocytes and RPE, internal organ defects and innate immunologic function, therefore phenocopying patients with Arthogryposis-Renal dysfunction-Cholestasis syndrome, Chediak-Higashi syndrome, Hermansky-Pudlak syndrome, and Griscelli syndrome.

In the RPE, intracellular trafficking is required for receptor-mediated phagocytosis and degradation of disc membranes shed from the apical tips of photoreceptor outer segments in a diurnal rhythm, and is essential for long-term viability and functionality of photoreceptors. Molecular analysis of the vps39 mutant confirmed defects in vision as a consequence of increased RPE cell size, fewer immature melanosomes and an accumulation of vesicles containing phagocytosed photoreceptor cell outer segments, consistent with a defect in the fusion of endocytic vesicles with lysosomes, supporting a role for VPS39 in the aetiology of human pathologies associated with defective intracellular trafficking. ${ }^{70}$

\section{Choroideremia}

The small GTP-binding protein rab escort protein 1 (REP1) is involved in the control of intracellular transport. REP1 binds newly synthesised Rab proteins and facilitates the addition of geranyl-geranyl groups, a posttranslational modification which is essential for Rab function in the regulation of intracellular trafficking in the $\mathrm{RPE}$ and photoreceptors. Choroideremia (CHM) is a progressive chorioretinal dystrophy caused by mutations in the CHM gene, which encodes REP1. Loss-of-function of REP1 causes defective prenylation of a subset of Rab proteins with subsequent disruption of intracellular trafficking leading to a progressive degeneration of the choroid, RPE and photoreceptors. Zebrafish carrying a recessive nonsense mutation in chm initially exhibit areas of RPE hypertrophy and atrophy in the periphery followed by progressive cell death in the RPE and peripheral retina which leads to severe loss of retinal lamination and degeneration throughout, consistent with CHM patients (Figure 3). ${ }^{21}$ 

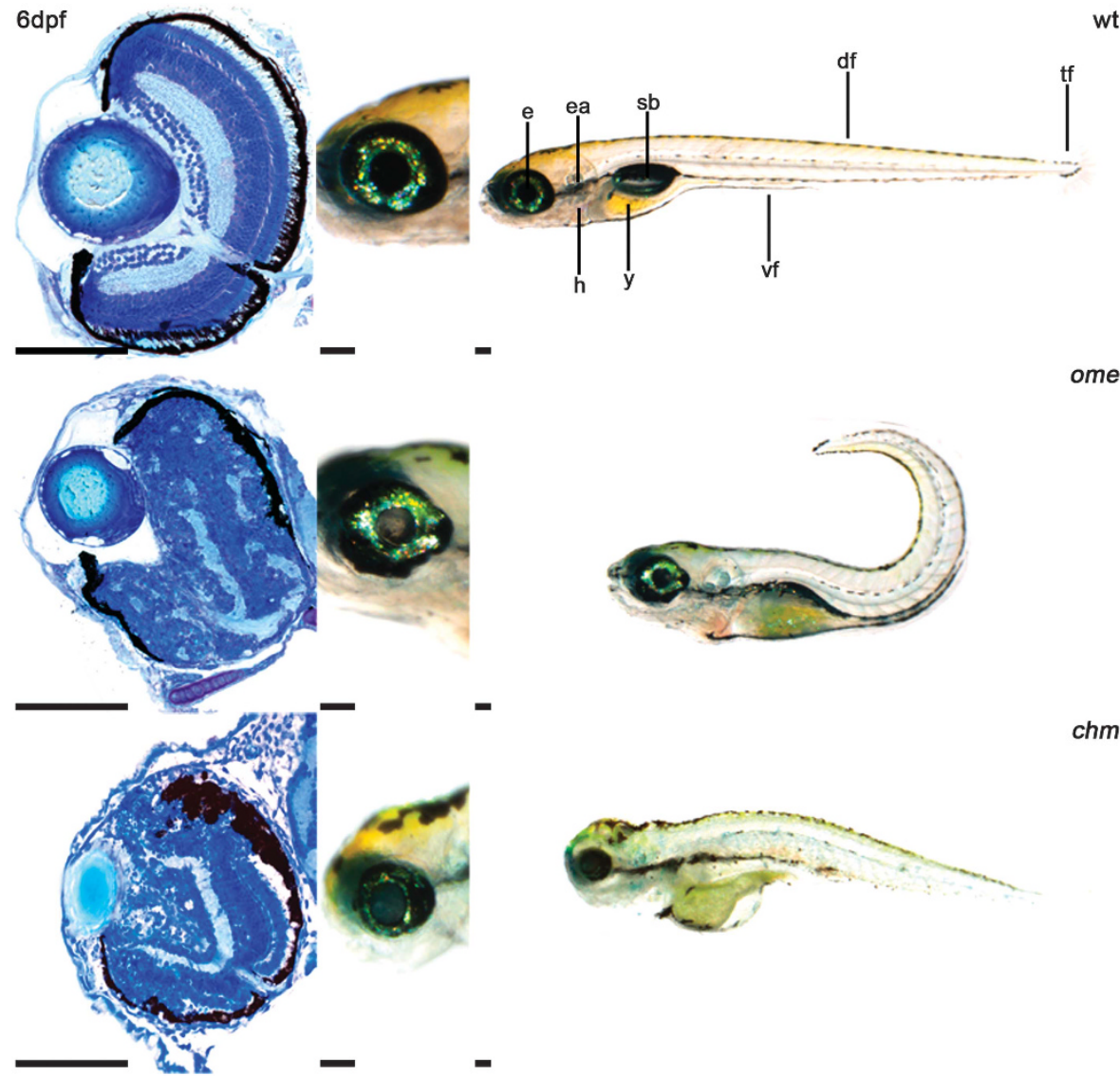

ome

chm

Figure 3 Gross morphology and retinal histology of wild-type (wt), ome and chm mutants at 6 d.p.f. Left panel: coronal retinal sections. The wild-type retina shows characteristic stratification in three nuclear and two plexiform layers. The ome $\left(\mathrm{crb} 2 a^{289 / 289}\right)$ mutant shows widespread retinal degeneration with loss of lamination and irregular patchy islands of plexiform tissue, with absence of large areas of RPE. In $\mathrm{chm}\left(\mathrm{chm}^{r \mathrm{u} 848 / \mathrm{ru} 848}\right)$ mutant embryos, there is a hard compacted cataractous lens, extensive retinal degeneration with pyknotic nuclei, areas of photoreceptor cell loss, and RPE hypertrophy and atrophy. Middle and right panel: bright-field images showing whole eye and fish morphology. The ome mutant shows irregular curvature of the spine, cardiac oedema, persistent yolk sac, and absent swimbladder. The chm mutant displays a smaller eye size with a cataract lens, gross morphological abnormalities include cardiac and abdominal oedema, persistent yolk sac, absent swimbladder, reduced body length and curvature of the spine. e, eye; ea, ear; h, heart; $\mathrm{y}$, yolk sac; sb, swimbladder, df, dorsal fin; tf, tail fin. Scale bars $=100 \mu \mathrm{m}$.

The absence of REP1 in CHM, is compensated for by the homologous protein REP2, which is coded for by the intronless gene CHML (choroideremia-like), thought to arise from a retrogene insertion of the REP1 mRNA transcript during vertebrate evolution. The $\mathrm{chm}$ mutant zebrafish only has one rep isoform, therefore lacking any compensatory function resulting in early lethality at 5 d.p.f. as a consequence of multisystemic organ failure. ${ }^{71}$ It has been hypothesised that the maternal supply of rep1 stored in the embryonic yolk sac allows for sufficient Rab function to ensure proper intracellular cycling up to 4 d.p.f., thereafter the disease phenotype manifests. ${ }^{71}$ The $\mathrm{chm}$ mutant model provides a useful tool to characterise therapies aimed at boosting REP1 activity, for example novel drug classes that facilitate readthrough of nonsense mutations. $^{21}$

\section{Lebers congenital amaurosis}

Lebers congenital amaurosis (LCA) is a group of inherited severe early-onset retinal dystrophies with clinical and genetic heterogeneity. Currently at least 15 genes have been linked to LCA including CEP290, RPE65, CRB1, KCNJ13, GUCY2D, AIPL1, CRX, IMPDH1, LCA5, LRAT, RD3, RDH12, RPGRIP1, SPATA7, and TULP1.

Knockdown of the zebrafish orthologue $g u c y 2 f$ results in early visual dysfunction with visible outer segment and photoreceptor layer loss. ${ }^{72}$ Morpholino-induced knockdown of centrosomal protein $290 \mathrm{kDa}$ (cep290) resulted in delayed intracellular transport and reduced visual acuity despite a fully laminated retina, consistent with human LCA patients. ${ }^{73}$ Importantly, injection of cep290 mutants with an $N$-terminal CEP290 construct rescued visual function, supporting a potential treatment 
for LCA patients. Mutations in the zebrafish orthologue crb1 result in severe corneal defects ${ }^{74}$ which, in comparison, appear more pronounced than changes in the human LCA cornea. Conversely, the oko meduzy (ome) zebrafish mutant which harbours mutations in crb2a, exhibits neuronal patterning defects of the retina, preceded by reduced neuroepithelial cell integrity more consistent with human CRB1-related LCA (Figure 3). ${ }^{75}$ The rpe65a-deficient zebrafish also exhibits changes in retinal physiology, presenting with shortened, deteriorating rod outer segments which interfere with photoreceptor functionality. ${ }^{76}$

\section{Retinitis pigmentosa}

Retinitis pigmentosa (RP) is a class of diseases that leads to progressive retinal degeneration characterised by dysfunction of the photoreceptors with retinal vessel attenuation, and progressive cell death.

X-linked RP is one of the most severe forms of RP, characterised by early-onset and rapid progression of vision loss before the fourth decade. Multiple X-linked RP causative genetic loci have been mapped on the $\mathrm{X}$-chromosome, although mutations in retinitis pigmentosa 2 (RP2) and retinitis pigmentosa GTPase regulator (RPGR) genes account for the vast majority of all $\mathrm{X}$-linked RP cases.

Morpholino-induced knockdown of the RP2 zebrafish orthologue rp2 results in microphthalmia, defective retinal lamination, and abnormal photoreceptor morphology including lack of outer segments with extensive retinal cell death and retinal degeneration, consistent with features of human X-linked RP. ${ }^{77}$ The $r p g r$ zebrafish morphant presents with a similar retinal phenotype as a consequence of cell death in the dysplastic retina. ${ }^{78}$ These zebrafish morphant phenotypes can be rescued by injection of human $R P 2$ or RPGR mRNA, respectively, indicative of a functional role for $R P 2$ and $R P G R$ in the pathogenesis of human X-linked RP. ${ }^{78}$

Mutations in the Rhodopsin (RHO) gene are the most common cause of human autosomal dominant RP. Rhodopsin is a member of the G-protein-coupled receptor family and has a role in phototransduction in rod photoreceptors. Transgenic fish with the human rhodopsin Q344X mutation under the control of the zebrafish rhodopsin promoter show photoreceptor degeneration as a consequence of increased apoptotic cell death. Importantly, cone photoreceptor number remained unchanged in the transgenic zebrafish retina, therefore mimicking human RP with the $\mathrm{RHO}$ mutation. ${ }^{79}$

Mutations in ceramide kinase-like gene (CERKL) have been associated with the severe retinal degeneration described in patients with RP26. The zebrafish cerkl protein protects retinal cells from oxidative stress-induced apoptosis. Morpholino-induced knockdown of the zebrafish cerkl resulted in increased retinal cell death with rod and cone photoreceptor degeneration. ${ }^{80}$ In addition, mutations in c2orf 71 , the zebrafish orthologue of C2ORF71 (responsible for human RP54), resulted in shortened photoreceptor outer segments, and attenuated visual response to light exposure. ${ }^{81}$ The ability to simulate discrete phenotypes associated with human RP in zebrafish models such as these, has proved invaluable in confirming human genes as causative for RP.

$\mathrm{RP}$ can also occur in association with systemic disease like in the genetically heterogeneous ciliopathy Bardet-Biedl syndrome, which is characterised by RP, obesity, kidney dysfunction, polydactyly, behavioural dysfunction, and hypogonadism. The zebrafish photoreceptor mutant oval (ovl) which encodes the cilium gene, IFT88 displays loss of outer segments due to mislocalised visual pigment and cilia dysfunction, similar to the mislocalisation observed in human photoreceptor cell death caused by RP, suggesting that ectopic phototransduction may have an important role in photoreceptor cell death. ${ }^{82}$

Usher syndrome (USH) is an autosomal recessive genetic disease characterised by combined hearing and vision loss and occasionally balance problems. USH is classified into three subtypes according to clinical severity and symptoms; Usher type 1 (USH1) presents as severe to profound congenital sensorineural deafness and vestibular areflexia with onset of RP within the first decade of life; type 2 (USH2) patients show moderate to severe hearing loss, normal vestibular function and pre-/post-pubertal onset of RP; and type 3 (USH3) patients have progressive hearing loss, sporadic vestibular dysfunction, and variable onset of RP. The exact function of USH proteins remains contentious, however, the identification of multiple USH-causative genes and their analysis in zebrafish models suggests that compromised photoreceptors, RPE cells and Müller cells may all underlie the cellular pathogenesis of USHassociated RP.

The gene MYO7A encodes human myosin VIIA, a protein responsible for USH1B, is expressed in RPE and photoreceptor cells of the human retina. Homozygous mutations in zebrafish myo7aa resulted in mild retinal degeneration by 10 d.p.f.; mutants presented with elevated cell death in the outer nuclear layer of the retina and photoreceptor degeneration. ${ }^{83}$ A number of large acellular holes were observed in the RPE of myo7aa mutants following light damage by exposure to constant light conditions, consistent with an inability to clear outer segment debris and subsequent non-autonomous RPE degeneration.

USH1C encodes the PDZ-domain-containing protein harmonin. The harmonin (ush1c) mutant presented with 
defective photoreceptor function attributed to a primary defect in Müller glial cells and subsequent ribbon synapse stability and function. ${ }^{84}$ Interestingly, PDZ domaincontaining 7 (PDZD7) encodes a ciliary protein with homology to the USH1C and USH2D proteins. Although morpholino-induced knockdown of $u \operatorname{sh} 2 a$ or $p d z d 7 a$ alone resulted in moderate levels of photoreceptor cell death in the retina, combined morpholino-induced partial ush $2 a$; $p d z d 7 a$, or $p d z d 7 a ; g p r 98$ knockdown exacerbated photoreceptor death, consistent with the possibility of human digenic inheritance of USH-associated mutations and retinal disease modifiers in patients with USH2A. ${ }^{85}$

Point mutations and large deletions in PCDH15 are the cause of human USH1. ${ }^{86,87}$ Reduction in the zebrafish orthologue $p c d h 15 b$ resulted in short and disorganised outer segments that lack interdigitation with the RPE. ${ }^{88}$ This early-onset photoreceptor malformation is likely attributed to progressive photoreceptor death as a consequence of impaired contact between the RPE and outer segments, suggesting a role for PCDH15 in maintaining the structural integrity of the photoreceptor outer segment.

\section{Vascular disease}

Mutations in plexin D1 (plxnd1) in the zebrafish mutant out of bounds (obd) result in patterning defects of intersegmental vessels and increased primary branching of the hyaloid vessels, providing a model to explore the mechanisms that govern normal, and aberrant human retinal angiogenesis. ${ }^{89}$ Importantly, zebrafish obd mutants survive to adulthood unlike knock-out mice, enabling characterisation of the hyaloid vasculature.

In addition, zebrafish with mutations in the von HippelLindau (VHL) orthologue, vhl present with increased choroidal and hyaloid vascular networks and display a systemic hypoxic response, including vascular leakage in the retina, lesions throughout the retinal layers, and retinal detachment of the retinal nerve layers from the RPE, consistent with VHL patients that develop retinal neovascularisation. ${ }^{90}$ Importantly, overproduction of hypoxia-induced mRNAs is a hallmark of highly vascularised neoplasms associated with inactivation of the VHL tumour suppressor gene in human retinal haemangioblastomas, highlighting the clinical relevance of the zebrafish model for the study of hypoxia-induced pathological angiogenesis.

Familial exudative vitreoretinopathy (FEVR) is characterised by abnormal retinal angiogenesis, resulting in retinal detachment and sight impairment. Mutations in zinc finger protein 408 (ZNF408) have been associated with autosomal dominant FEVR. Morpholino-induced knockdown of zebrafish znf408 resulted in defects in retinal radial vessel sprouting, highlighting a functional link between znf408 and retinal blood vessel formation. ${ }^{91}$

\section{Therapies for ocular disorders}

Gene therapy approaches represent the most promising therapeutic option for the treatment of genetic eye disease; the routes of administration are local and systemic for ocular diseases. Vectors derived from adenoassociated virus (AAV) are most frequently used for ocular gene delivery due their small size and ability to efficiently transduce retinal cell types in vivo.

Although viral gene transfer in zebrafish has been achieved using multiple retroviral integrases, significant drawbacks have included toxicity, complexity of virus production and modification, and difficulty in achieving high titres. As an alternative, effective gene delivery and expression using the Sleeping Beauty transposon system has been described. ${ }^{92}$ Injection of a transposon construct containing GFP under control of a lens-specific expression cassette from the $\gamma$-crystallin gene produced embryos with eye-specific GFP expression. Similarly, the Tol2 transposon-based vector system can drive overexpression of $c r b 3 a$ in the zebrafish otic vesicle under the control of the heat-shock promoter. ${ }^{93}$ Induction of $c r b 3 a$ expression by heat exposure resulted in profound epithelial polarity defects limited to the retinal neuroepithelium, and disorganised retinal architecture similar to $c r b 2 a$ mutants providing proof-of-concept for vector-based and tissuespecific gene delivery. Established vector-based gene delivery also provides an opportunity to carry out conditional phenotypic rescue experiments applicable against zebrafish mutants and morphants, representing a powerful tool for high-throughput drug screening.

\section{Small-molecule drugs}

Pharmacological screens in zebrafish can be used to identify small-molecule drugs that affect biological processes, by perturbing protein function. Although little precedent exists for small-molecule screens focusing on zebrafish retinal development, this approach has been successfully employed to investigate other zebrafish organs and behaviours. Recently, a quantitative wholeorganism screening method combining high-throughput screening instrumentation with reporter-based assays was developed to identify compounds that elevated insulin reporter activity. ${ }^{94}$ Over 500000 zebrafish embryos were screened and 177 drugs implicated as candidates to treat $\beta$-cell paucity in diabetic patients. Importantly, a proportion of these candidates were already approved for use in humans highlighting the plausibility of rapid clinical translation of such screening approaches. 
Current therapies for delaying defective ocular angiogenesis include laser surgery or molecular inhibition of pro-angiogenic factors. The small-molecule drug LY294002, a PI3 kinase inhibitor, was identified as a selective inhibitor of both developmental and ectopic hyaloid angiogenesis in the eye in a targeted screen of known regulators of angiogenesis using zebrafish. Intraocular injection inhibited ocular angiogenesis without secondary systemic effects or threatening visual function, demonstrating the potential to effectively, and safely treat unwanted neovascularisation in eye disease with isoform-specific inhibitors or vascular-targeted prodrugs as monotherapies or part of combination angiostatic approaches. ${ }^{95}$ Similarly, a random pharmacological screen involved treatment of zebrafish with 2000 small molecules from The Spectrum library (Microsource Discovery Systems, Inc., Gaylordsville, CT, USA) to identify compounds affecting retinal vasculature that could be of therapeutic importance. ${ }^{96}$ Compounds which caused collapse and loss of retinal vessels, or increased vessel diameter were identified, with the potential to alleviate symptoms of persistent hyperplastic foetal vasculature or retinal blood vessel occlusion, respectively.

Nonsense mutations that introduce premature stop codons account for $\sim 30 \%$ of genetic eye disease. Molecular therapies that target premature stop codons offer a practical treatment option. PAX2 nonsense mutations have been identified in ocular coloboma. The no isthmus (noi ${ }^{\text {tu29a }}$ ) zebrafish mutant has a recessive nonsense mutation in pax 2.1 and the grumpy $\left(\right.$ gup $\left.^{m 189}\right)$ zebrafish mutant has a recessive nonsense mutation in lamb1, both resulting in optic fissure closure defects. Mutant noi and gup larvae dosed with aminoglycosides, gentamicin, and paromomycin, demonstrated complete fusion of the optic fissure and regular retinal lamination by 9 d.p.f. ${ }^{21}$ The choroideremia mutant zebrafish $\left(\mathrm{Chm}^{\text {rus48 }}\right)$ has a recessive nonsense mutation in the second exon of the chm gene, which manifests with severe retinal degeneration, microphthalmia, and cataract. ${ }^{71}$ Treatment with gentamicin or paromomycin resulted in normalisation of retinal lamination with no signs of chorioretinal degeneration at $9 \mathrm{~d}$. p.f. ${ }^{21}$ highlighting the applicability of pharmacological agents to readthrough premature stop codons in the treatment of inherited eye disorders caused by nonsense mutations.

\section{Molecular therapies; targeting molecular pathways for therapeutic intervention}

Reverse genetics has proved to be a powerful way to delineate roles of canonical signalling pathways involved in zebrafish ocular development and disease. Ocular coloboma is associated with increased apoptotic activity at the site of defective optic fissure fusion. ${ }^{21}$ Curcumin (chemical structure diferuloylmethane) has a wide range of pharmacological effects, including anti-apoptotic by inhibition of caspase-3 expression. zVAD-FMK (fluoromethyl ketone molecule) is a specific cellpermeable pan-caspase inhibitor. Treatment of the gup coloboma mutant with curcumin or zVAD-FMK resulted in a reduction in apoptotic activity and a milder coloboma phenotype. ${ }^{97}$ In addition, inhibition of RIP1, a regulatory molecule of necroptosis and apoptosis, with the smallmolecule drug necrostatin-1, rescued the colobomatous defect in pax2.1-deficient zebrafish mutants. Future therapeutic strategies may be based on small-molecule drugs that bypass the gene defects causing common congenital tissue fusion defects. ${ }^{20}$

\section{Photoreceptor regeneration}

The adult fish retina possesses a robust capacity to replace lost neurons following injury. Several lesion paradigms have been employed to investigate zebrafish retinal regeneration including light lesions which specifically destroy photoreceptors, intravitreal injection of ouabain neurotoxin which destroys ganglion cells and the inner nuclear layer, and physical lesions causing localised retinal damage. In all cases, lost retinal neurons were regenerated, retinal lamination normalised, and visual function restored.

Although a number of genetic, molecular, and cell biological techniques have proven invaluable in understanding mechanisms that underpin zebrafish retinal degeneration and regeneration, most modalities are unable to generate a dynamic retinal picture during this neuronal cell death and regeneration. Optical coherence tomography (OCT) is a non-invasive imaging technique essential in diagnosing and monitoring human retinal disease. Research has demonstrated a good correlation between representative OCT retinal layers and retinal histopathology in humans, facilitating the ability to discern important features of anterior and posterior segments of the eye. Importantly, OCT has also been employed to assay dynamic morphological changes during zebrafish retinal lamination and photoreceptor loss, and regeneration with light-induced or ouabaininduced damage in vivo. ${ }^{98}$ It is possible to not only resolve individual retinal layers, from the retinal nerve fibre layer to the RPE, but also the photoreceptor mosaic, emphasising the high-resolution imaging capacity of OCT in small-animal imaging (Figure 4). This work potentiates the ability to study dynamic processes of zebrafish retinal development, damage, and regeneration whilst providing a means to characterise retinal degenerative disorders and assess the efficacy of potential treatments.

The efficacy of pathway-specific compounds has been investigated in zebrafish retinal regeneration models. 

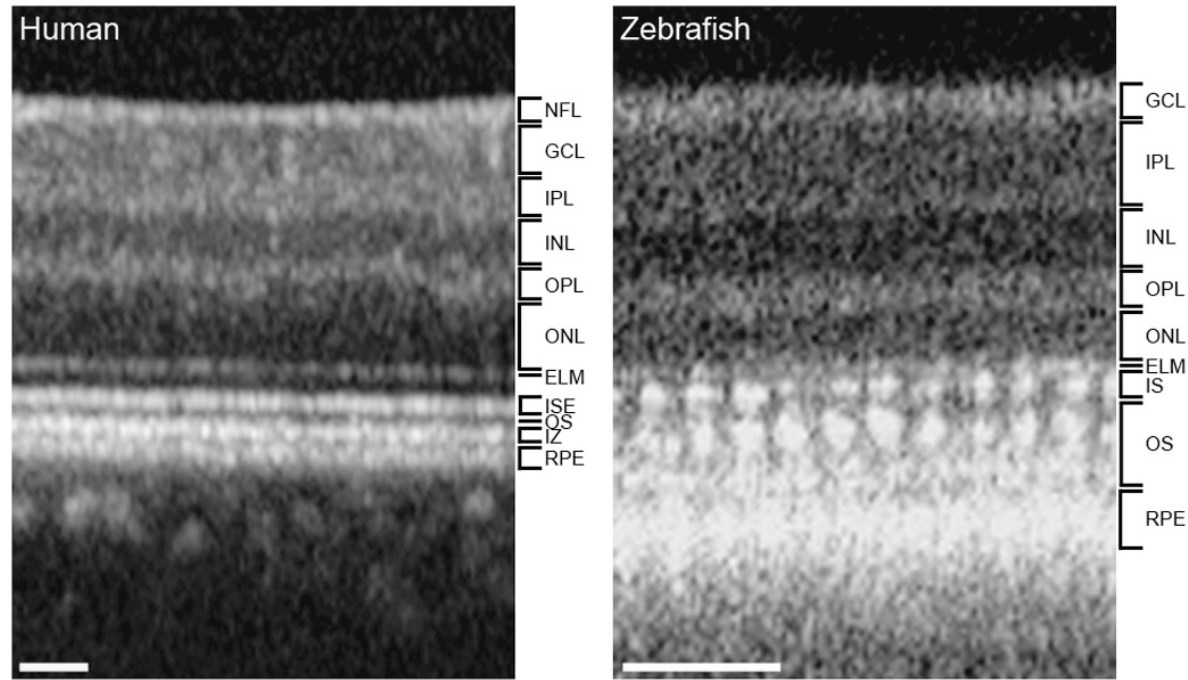

Figure 4 In vivo OCT horizontal scans of the human and zebrafish retina. In the human OCT image, the site of the IS/OS border aligns with the ellipsoids of the IS, therefore referred to as the IS ellipsoid (ISE) band. The interdigitation zone corresponds to the configuration of the RPE apices with the photoreceptor outer segments. Standard resolution OCT imaging allows the identification of individual photoreceptor inner and outer segments in the living zebrafish retina. NFL, nerve fibre layer; GCL, ganglion cell layer; IPL, inner plexiform layer; INL, inner nuclear layer; OPL, outer plexiform layer; ONL, outer nuclear layer; ELM, external limiting membrane; ISE, inner segment ellipsoid; OS, outer segment; IZ, interdigitation zone; RPE, retinal pigment epithelium. Scale bars $=200 \mu \mathrm{m}$.

$\beta$-Catenin/Wnt signalling has been implicated in Müllerglial proliferation in the regenerating adult zebrafish retina. ${ }^{99}$ Following retinal injury, $\beta$-catenin accumulates in the nucleus of the Müller glial-derived progenitors. The tankyrase inhibitor, XAV939, selectively inhibits $\beta$-catenin-mediated transcription and pyrvinium, a casein kinase 1- $\alpha$ activator, promotes $\beta$-catenin degradation. Blocking $\beta$-catenin accumulation by injection of XAV939 or pyrvinium at the time of retinal injury resulted in reduced proliferation of Müller glial-derived progenitors indicating that $\beta$-catenin is required for retinal progenitor cell proliferation. ${ }^{100}$ Importantly, $\beta$-catenin signalling can be enhanced by injection of the GSK-3 $\beta$-inhibitor, lithium chloride in injured and uninjured zebrafish retinas, stimulating Müller glial dedifferentiation and proliferation into multiple retinal neuronal subtypes. ${ }^{100}$

Reprogramming of endogenous Müller glial cells to a stem cell function, or transplantation of stem cells or progenitor cells into diseased retinas may provide therapeutic potential for degenerative retinal disorders. Inspiration for Müller-cell therapies comes from findings that suggest Müller glia function as multipotent retinal stem cells, which can generate retinal neurons in response to loss of photoreceptors in the differentiated zebrafish retina. Mammalian Müller glia also exhibit some neurogenic properties indicative of an ability to regenerate retinal neurons. Elucidating the specific properties of zebrafish Müller glia that facilitate their innate capacity to regenerate retinal neurons and the identification of chemical modulators of this process will provide invaluable information to harness alternative therapies to treat human retinal degeneration.

\section{Summary}

The zebrafish has emerged as a robust model system for studying cellular and molecular mechanisms that underpin normal ocular development and human eye disease. Largescale genetic screens have allowed the identification of candidate genes, which provide a deeper understanding of human pathogenicity and support the development of potential therapies to combat untreatable genetic disorders. The development of reliable zebrafish models of eye disease through technologies such as CRISPR, coupled with more sophisticated transgenic approaches to visualise cellular processes in vivo, support the expanded use of the zebrafish as a tool to functionally annotate human ocular disease alleles. Ongoing work to dissect the signalling pathways that converge to regulate ocular development will not only infer the pathophysiological processes that underlie complex ocular diseases, but also facilitate the development of geneand cell-mediated therapeutic strategies for their treatment and prevention.

\section{Conflict of interest}

The authors declare no conflict of interest. 


\section{Acknowledgements}

We gratefully acknowledge funding from Fight for Sight UK, Moorfields Eye Charity, the Choroideremia Research Foundation, the Academy of Medical Sciences and the NIHR Biomedical Research Centre at Moorfields Eye Hospital and UCL Institute of Ophthalmology. Thanks to Philip Luthert for providing the human retinal histology section and to Adam Dubis for his guidance with OCT imaging.

\section{References}

1 Howe K, Clark MD, Torroja CF, Torrance J, Berthelot C, Muffato $\mathrm{M}$ et al. The zebrafish reference genome sequence and its relationship to the human genome. Nature 2013; 496 (7446): 498-503.

2 Schmitt EA, Dowling JE. Early eye morphogenesis in the zebrafish, Brachydanio rerio. J Comp Neurol 1994; 344(4): 532-542.

3 Schmitt EA, Dowling JE. Early retinal development in the zebrafish, Danio rerio: light and electron microscopic analyses. J Comp Neurol 1999; 404(4): 515-536.

4 Barishak YR. Embryology of the eye and its adnexae. Dev Ophthalmol 1992; 24: 1-142.

5 Greiling TM, Aose M, Clark JI. Cell fate and differentiation of the developing ocular lens. Invest Ophthalmol Vis Sci 2010; 51(3): 1540-1546.

6 Zhao XC, Yee RW, Norcom E, Burgess H, Avanesov AS, Barrish JP et al. The zebrafish cornea: structure and development. Invest Ophthalmol Vis Sci 2006; 47(10): 4341-4348.

7 Schmitt EA, Dowling JE. Comparison of topographical patterns of ganglion and photoreceptor cell differentiation in the retina of the zebrafish, Danio rerio. I Comp Neurol 1996; 371(2): 222-234.

8 Easter Jr SS, Nicola GN. The development of vision in the zebrafish (Danio rerio). Dev Biol 1996; 180(2): 646-663.

9 Bilotta J, Saszik S, Sutherland SE. Rod contributions to the electroretinogram of the dark-adapted developing zebrafish. Dev Dyn 2001; 222(4): 564-570.

10 Malicki J, Neuhauss SC, Schier AF, Solnica-Krezel L, Stemple DL, Stainier DY et al. Mutations affecting development of the zebrafish retina. Development 1996; 123: 263-273.

11 Clark KJ, Urban MD, Skuster KJ, Ekker SC. Transgenic zebrafish using transposable elements. Methods Cell Biol 2011; 104: 137-149.

12 Tallafuss A, Gibson D, Morcos P, Li Y, Seredick S, Eisen J et al. Turning gene function $\mathrm{ON}$ and OFF using sense and antisense photo-morpholinos in zebrafish. Development 2012; 139(9): 1691-1699.

13 Andrews OE, Cha DJ, Wei C, Patton JG. RNAi-mediated gene silencing in zebrafish triggered by convergent transcription. Sci Rep 2014; 4: 5222.

14 Hwang WY, Peterson RT, Yeh JR. Methods for targeted mutagenesis in zebrafish using TALENs. Methods 2014; 69(1): 76-84.

15 Dahlem TJ, Hoshijima K, Jurynec MJ, Gunther D, Starker CG, Locke AS et al. Simple methods for generating and detecting locus-specific mutations induced with TALENs in the zebrafish genome. PLoS Genet 2012; 8(8): e1002861.
16 Shah AN, Davey CF, Whitebirch AC, Miller AC, Moens CB. Rapid reverse genetic screening using CRISPR in zebrafish. Nat Methods 2015; 12(6): 535-540.

17 Gregory-Evans CY, Williams MJ, Halford S, Gregory-Evans K. Ocular coloboma: a reassessment in the age of molecular neuroscience. J Med Genet 2004; 41(12): 881-891.

18 Bower M, Salomon R, Allanson J, Antignac C, Benedicenti F, Benetti E et al. Update of PAX2 mutations in renal coloboma syndrome and establishment of a locus-specific database. Hum Mutat 2012; 33(3): 457-466.

19 Lun K, Brand M. A series of no isthmus (noi) alleles of the zebrafish pax2.1 gene reveals multiple signaling events in development of the midbrain-hindbrain boundary. Development 1998; 125(16): 3049-3062.

20 Viringipurampeer IA, Ferreira T, DeMaria S, Yoon JJ, Shan X, Moosajee $\mathrm{M}$ et al. Pax2 regulates a fadd-dependent molecular switch that drives tissue fusion during eye development. Hum Mol Genet 2012; 21(10): 2357-2369.

21 Moosajee M, Gregory-Evans K, Ellis CD, Seabra MC, Gregory-Evans CY. Translational bypass of nonsense mutations in zebrafish rep1, pax2.1 and lamb1 highlights a viable therapeutic option for untreatable genetic eye disease. Hum Mol Genet 2008; 17(24): 3987-4000.

22 Gross JM, Perkins BD, Amsterdam A, Egana A, Darland T, Matsui JI et al. Identification of zebrafish insertional mutants with defects in visual system development and function. Genetics 2005; 170(1): 245-261.

23 French CR, Stach TR, March LD, Lehmann OJ, Waskiewicz AJ. Apoptotic and proliferative defects characterize ocular development in a microphthalmic BMP model. Invest Ophthalmol Vis Sci 2013; 54(7): 4636-4647.

24 Nadauld LD, Chidester S, Shelton DN, Rai K, Broadbent T, Sandoval IT et al. Dual roles for adenomatous polyposis coli in regulating retinoic acid biosynthesis and Wnt during ocular development. Proc Natl Acad Sci USA 2006; 103(36): 13409-13414.

25 Lee J, Gross JM. Laminin beta1 and gamma1 containing laminins are essential for basement membrane integrity in the zebrafish eye. Invest Ophthalmol Vis Sci 2007; 48(6): 2483-2490.

26 Gerth-Kahlert C, Williamson K, Ansari M, Rainger JK, Hingst V, Zimmermann $\mathrm{T}$ et al. Clinical and mutation analysis of 51 probands with anophthalmia and/or severe microphthalmia from a single center. Mol Genet Genomic Med 2013; 1(1): 15-31.

27 Asai-Coakwell M, French CR, Berry KM, Ye M, Koss R, Somerville $\mathrm{M}$ et al. GDF6, a novel locus for a spectrum of ocular developmental anomalies. Am J Hum Genet 2007; 80(2): 306-315.

28 Gosse NJ, Baier H. An essential role for Radar (Gdf6a) in inducing dorsal fate in the zebrafish retina. Proc Natl Acad Sci USA 2009; 106(7): 2236-2241.

29 den Hollander AI, Biyanwila J, Kovach P, Bardakjian T, Traboulsi EI, Ragge NK et al. Genetic defects of GDF6 in the zebrafish out of sight mutant and in human eye developmental anomalies. BMC Genet 2010; 11: 102.

30 Nelson SM, Park L, Stenkamp DL. Retinal homeobox 1 is required for retinal neurogenesis and photoreceptor differentiation in embryonic zebrafish. Dev Biol 2009; 328(1): 24-39.

31 Yin J, Morrissey ME, Shine L, Kennedy C, Higgins DG, Kennedy BN. Genes and signaling networks regulated during zebrafish optic vesicle morphogenesis. BMC Genomics 2014; 15: 825. 
32 Kleinjan DA, Bancewicz RM, Gautier P, Dahm R, Schonthaler HB, Damante G et al. Subfunctionalization of duplicated zebrafish pax6 genes by cis-regulatory divergence. PLoS Genet 2008; 4(2): e29.

33 Coutinho P, Pavlou S, Bhatia S, Chalmers KJ, Kleinjan DA, van Heyningen V. Discovery and assessment of conserved Pax6 target genes and enhancers. Genome Res 2011; 21(8): 1349-1359.

34 Suzuki KT, Isoyama Y, Kashiwagi K, Sakuma T, Ochiai H, Sakamoto $\mathrm{N}$ et al. High efficiency TALENs enable F0 functional analysis by targeted gene disruption in Xenopus laevis embryos. Biol Open 2013; 2(5): 448-452.

35 Nasevicius A, Ekker SC. Effective targeted gene 'knockdown' in zebrafish. Nat Genet 2000; 26(2): 216-220.

36 Dougan ST, Warga RM, Kane DA, Schier AF, Talbot WS. The role of the zebrafish nodal-related genes squint and cyclops in patterning of mesendoderm. Development 2003; 130(9): 1837-1851.

37 Zhang J, Talbot WS, Schier AF. Positional cloning identifies zebrafish one-eyed pinhead as a permissive EGF-related ligand required during gastrulation. Cell 1998; 92(2): 241-251.

38 Lim S, Wang Y, Yu X, Huang Y, Featherstone MS, Sampath K. A simple strategy for heritable chromosomal deletions in zebrafish via the combinatorial action of targeting nucleases. Genome Biol 2013; 14(7): R69.

39 Chen CC, Yeh LK, Liu CY, Kao WW, Samples JR, Lin SJ et al. Morphological differences between the trabecular meshworks of zebrafish and mammals. Curr Eye Res 2008; 33(1): 59-72.

40 Gray MP, Smith RS, Soules KA, John SW, Link BA. The aqueous humor outflow pathway of zebrafish. Invest Ophthalmol Vis Sci 2009; 50(4): 1515-1521.

41 Link BA, Gray MP, Smith RS, John SW. Intraocular pressure in zebrafish: comparison of inbred strains and identification of a reduced melanin mutant with raised IOP. Invest Ophthalmol Vis Sci 2004; 45(12): 4415-4422.

42 Veth KN, Willer JR, Collery RF, Gray MP, Willer GB, Wagner DS et al. Mutations in zebrafish lrp2 result in adult-onset ocular pathogenesis that models myopia and other risk factors for glaucoma. PLoS Genet 2011; 7(2): e1001310.

43 Sowden JC. Molecular and developmental mechanisms of anterior segment dysgenesis. Eye 2007; 21(10): 1310-1318.

44 McMahon C, Semina EV, Link BA. Using zebrafish to study the complex genetics of glaucoma. Comp Biochem Physiol C Toxicol Pharmacol 2004; 138(3): 343-350.

45 Berry FB, Skarie JM, Mirzayans F, Fortin Y, Hudson TJ, Raymond $\mathrm{V}$ et al. FOXC1 is required for cell viability and resistance to oxidative stress in the eye through the transcriptional regulation of FOXO1A. Hum Mol Genet 2008; 17(4): 490-505.

46 Skarie JM, Link BA. FoxC1 is essential for vascular basement membrane integrity and hyaloid vessel morphogenesis. Invest Ophthalmol Vis Sci 2009; 50(11): 5026-5034.

47 Skarie JM, Link BA. The primary open-angle glaucoma gene WDR36 functions in ribosomal RNA processing and interacts with the p53 stress-response pathway. Hum Mol Genet 2008; 17(16): 2474-2485.

48 Park S, Jamshidi Y, Vaideanu D, Bitner-Glindzicz M, Fraser S, Sowden JC. Genetic risk for primary open-angle glaucoma determined by LMX1B haplotypes. Invest Ophthalmol Vis Sci 2009; 50(4): 1522-1530.
49 McMahon C, Gestri G, Wilson SW, Link BA. Lmx1b is essential for survival of periocular mesenchymal cells and influences Fgf-mediated retinal patterning in zebrafish. Dev Biol 2009; 332(2): 287-298.

50 Klintworth GK. Corneal dystrophies. Orphanet J Rare Dis 2009; 4 : 7.

51 Boisset G, Polok BK, Schorderet DF. Characterization of pip5k3 fleck corneal dystrophy-linked gene in zebrafish. Gene Expr Patterns 2008; 8(6): 404-410.

52 Pellegata NS, Dieguez-Lucena JL, Joensuu T, Lau S, Montgomery KT, Krahe R et al. Mutations in KERA, encoding keratocan, cause cornea plana. Nat Genet 2000; 25(1): 91-95.

53 Yeh LK, Liu CY, Chien CL, Converse RL, Kao WW, Chen MS et al. Molecular analysis and characterization of zebrafish keratocan (zKera) gene. J Biol Chem 2008; 283(1): 506-517.

54 Kulkarni BB, Tighe PJ, Mohammed I, Yeung AM, Powe DG, Hopkinson A et al. Comparative transcriptional profiling of the limbal epithelial crypt demonstrates its putative stem cell niche characteristics. BMC Genomics 2010; 11: 526.

55 Vereb Z, Albert R, Poliska S, Olstad OK, Akhtar S, Moe MC et al. Comparison of upstream regulators in human ex vivo cultured cornea limbal epithelial stem cells and differentiated corneal epithelial cells. BMC Genomics 2013; 14: 900.

56 Semina EV, Bosenko DV, Zinkevich NC, Soules KA, Hyde DR, Vihtelic TS et al. Mutations in laminin alpha 1 result in complex, lens-independent ocular phenotypes in zebrafish. Dev Biol 2006; 299(1): 63-77.

57 Shiels A, Hejtmancik JF. Molecular genetics of cataract. Prog Mol Biol Transl Sci 2015; 134: 203-218.

58 Vihtelic TS, Yamamoto Y, Springer SS, Jeffery WR, Hyde DR. Lens opacity and photoreceptor degeneration in the zebrafish lens opaque mutant. Dev Dyn 2005; 233(1): 52-65.

59 Goishi K, Shimizu A, Najarro G, Watanabe S, Rogers R, Zon LI et al. AlphaA-crystallin expression prevents gammacrystallin insolubility and cataract formation in the zebrafish cloche mutant lens. Development 2006; 133(13): 2585-2593.

60 Bredrup C, Matejas V, Barrow M, Blahova K, Bockenhauer D, Fowler DJ et al. Ophthalmological aspects of Pierson syndrome. Am J Ophthalmol 2008; 146(4): 602-611.

61 Pathania M, Semina EV, Duncan MK. Lens extrusion from laminin alpha 1 mutant zebrafish. ScientificWorldJournal 2014; 2014: 524929.

62 Hansen L, Comyn S, Mang Y, Lind-Thomsen A, Myhre L, Jean $\mathrm{F}$ et al. The myosin chaperone UNC45B is involved in lens development and autosomal dominant juvenile cataract. Eur J Hum Genet 2014; 22(11): 1290-1297.

63 Hingorani M, Hanson I, van Heyningen V. Aniridia. Eur J Hum Genet 2012; 20(10): 1011-1017.

64 Bhatia S, Bengani H, Fish M, Brown A, Divizia MT, de Marco R et al. Disruption of autoregulatory feedback by a mutation in a remote, ultraconserved PAX6 enhancer causes aniridia. Am J Hum Genet 2013; 93(6): 1126-1134.

65 Braasch I, Liedtke D, Volff JN, Schartl M. Pigmentary function and evolution of tyrp1 gene duplicates in fish. Pigment Cell Melanoma Res 2009; 22(6): 839-850.

66 Page-McCaw PS, Chung SC, Muto A, Roeser T, Staub W, Finger-Baier $\mathrm{KC}$ et al. Retinal network adaptation to bright light requires tyrosinase. Nat Neurosci 2004; 7(12): 1329-1336.

67 Beirl AJ, Linbo TH, Cobb MJ, Cooper CD. oca2 Regulation of chromatophore differentiation and number is cell type 
specific in zebrafish. Pigment Cell Melanoma Res 2014; 27(2): 178-189.

68 Tsetskhladze ZR, Canfield VA, Ang KC, Wentzel SM, Reid KP, Berg AS et al. Functional assessment of human coding mutations affecting skin pigmentation using zebrafish. PLoS One 2012; 7(10): e47398.

69 Gronskov K, Dooley CM, Ostergaard E, Kelsh RN, Hansen L, Levesque MP et al. Mutations in c10orf11, a melanocytedifferentiation gene, cause autosomal-recessive albinism. Am J Hum Genet 2013; 92(3): 415-421.

70 Schonthaler HB, Fleisch VC, Biehlmaier O, Makhankov Y, Rinner O, Bahadori R et al. The zebrafish mutant lbk/vam6 resembles human multisystemic disorders caused by aberrant trafficking of endosomal vesicles. Development 2008; 135(2): 387-399.

71 Moosajee M, Tulloch M, Baron RA, Gregory-Evans CY, Pereira-Leal JB, Seabra MC. Single choroideremia gene in nonmammalian vertebrates explains early embryonic lethality of the zebrafish model of choroideremia. Invest Ophthalmol Vis Sci 2009; 50(6): 3009-3016.

72 Stiebel-Kalish H, Reich E, Rainy N, Vatine G, Nisgav Y, Tovar A et al. Gucy2f zebrafish knockdown-a model for Gucy2d-related leber congenital amaurosis. Eur J Hum Genet 2012; 20(8): 884-889.

73 Baye LM, Patrinostro X, Swaminathan S, Beck JS, Zhang Y, Stone EM et al. The N-terminal region of centrosomal protein 290 (CEP290) restores vision in a zebrafish model of human blindness. Hum Mol Genet 2011; 20(8): 1467-1477.

74 Beyer J, Zhao XC, Yee R, Khaliq S, McMahon TT, Ying H et al. The role of crumbs genes in the vertebrate cornea. Invest Ophthalmol Vis Sci 2010; 51(9): 4549-4556.

75 Malicki J, Driever W. oko meduzy mutations affect neuronal patterning in the zebrafish retina and reveal cell-cell interactions of the retinal neuroepithelial sheet. Development 1999; 126(6): 1235-1246.

76 Schonthaler HB, Lampert JM, Isken A, Rinner O, Mader A, Gesemann $\mathrm{M}$ et al. Evidence for RPE65-independent vision in the cone-dominated zebrafish retina. Eur J Neurosci. 2007; 26(7): 1940-1949.

77 Shu X, Zeng Z, Gautier P, Lennon A, Gakovic M, Cheetham ME et al. Knockdown of the zebrafish ortholog of the retinitis pigmentosa 2 (RP2) gene results in retinal degeneration. Invest Ophthalmol Vis Sci 2011; 52(6): 2960-2966.

78 Shu X, Zeng Z, Gautier P, Lennon A, Gakovic M, Patton EE et al. Zebrafish Rpgr is required for normal retinal development and plays a role in dynein-based retrograde transport processes. Hum Mol Genet 2010; 19(4): 657-670.

79 Nakao T, Tsujikawa M, Notomi S, Ikeda Y, Nishida K. The role of mislocalized phototransduction in photoreceptor cell death of retinitis pigmentosa. PLoS One 2012; 7(4): e32472.

80 Li C, Wang L, Zhang J, Huang M, Wong F, Liu X et al. CERKL interacts with mitochondrial TRX2 and protects retinal cells from oxidative stress-induced apoptosis. Biochim Biophys Acta 2014; 1842(7): 1121-1129.

81 Nishimura DY, Baye LM, Perveen R, Searby CC, AvilaFernandez A, Pereiro I et al. Discovery and functional analysis of a retinitis pigmentosa gene, C2ORF71. Am J Hum Genet 2010; 86(5): 686-695.

82 Tsujikawa M, Malicki J. Intraflagellar transport genes are essential for differentiation and survival of vertebrate sensory neurons. Neuron 2004; 42(5): 703-716.

83 Wasfy MM, Matsui JI, Miller J, Dowling JE, Perkins BD. myosin 7aa $(-/-)$ mutant zebrafish show mild photoreceptor degeneration and reduced electroretinographic responses. Exp Eye Res 2014; 122: 65-76.

84 Phillips JB, Blanco-Sanchez B, Lentz JJ, Tallafuss A, Khanobdee K, Sampath $\mathrm{S}$ et al. Harmonin (Ush1c) is required in zebrafish Muller glial cells for photoreceptor synaptic development and function. Dis Model Mech 2011; 4(6): 786-800.

85 Ebermann I, Phillips JB, Liebau MC, Koenekoop RK, Schermer B, Lopez I et al. PDZD7 is a modifier of retinal disease and a contributor to digenic Usher syndrome. J Clin Invest 2010; 120(6): 1812-1823.

86 Ahmed ZM, Riazuddin S, Bernstein SL, Ahmed Z, Khan S, Griffith $\mathrm{AJ}$ et al. Mutations of the protocadherin gene PCDH15 cause Usher syndrome type 1 F. Am J Hum Genet 2001; 69(1): 25-34.

87 Aller E, Jaijo T, Garcia-Garcia G, Aparisi MJ, Blesa D, Diaz-Llopis $\mathrm{M}$ et al. Identification of large rearrangements of the PCDH15 gene by combined MLPA and a CGH: large duplications are responsible for Usher syndrome. Invest Ophthalmol Vis Sci 2010; 51(11): 5480-5485.

88 Seiler C, Finger-Baier KC, Rinner O, Makhankov YV, Schwarz H, Neuhauss SC et al. Duplicated genes with split functions: independent roles of protocadherin 15 orthologues in zebrafish hearing and vision. Development 2005; 132(3): 615-623.

89 Alvarez Y, Cederlund ML, Cottell DC, Bill BR, Ekker SC, Torres-Vazquez J et al. Genetic determinants of hyaloid and retinal vasculature in zebrafish. BMC Dev Biol 2007; 7: 114 .

90 van Rooijen E, Voest EE, Logister I, Bussmann J, Korving J, van Eeden FJ et al. von Hippel-Lindau tumor suppressor mutants faithfully model pathological hypoxia-driven angiogenesis and vascular retinopathies in zebrafish. Dis Model Mech 2010; 3(5-6): 343-353.

91 Collin RW, Nikopoulos K, Dona M, Gilissen C, Hoischen A, Boonstra FN et al. ZNF408 is mutated in familial exudative vitreoretinopathy and is crucial for the development of zebrafish retinal vasculature. Proc Natl Acad Sci USA 2013; 110(24): 9856-9861.

92 Davidson AE, Balciunas D, Mohn D, Shaffer J, Hermanson S, Sivasubbu $\mathrm{S}$ et al. Efficient gene delivery and gene expression in zebrafish using the Sleeping Beauty transposon. Dev Biol 2003; 263(2): 191-202.

93 Omori Y, Malicki J. oko meduzy and related crumbs genes are determinants of apical cell features in the vertebrate embryo. Curr Biol 2006; 16(10): 945-957.

94 Wang G, Rajpurohit SK, Delaspre F, Walker SL, White DT, Ceasrine A et al. First quantitative high-throughput screen in zebrafish identifies novel pathways for increasing pancreatic $\beta$-cell mass. Elife; e-pub ahead of print 28 July 2015; doi:10.7554/eLife.08261.

95 Alvarez Y, Astudillo O, Jensen L, Reynolds AL, Waghorne N, Brazil DP et al. Selective inhibition of retinal angiogenesis by targeting PI3 kinase. PLoS One 2009; 4(11): e7867.

96 Kitambi SS, McCulloch KJ, Peterson RT, Malicki JJ. Small molecule screen for compounds that affect vascular development in the zebrafish retina. Mech Dev 2009; 126(5-6): 464-477. 
97 Gregory-Evans CY, Moosajee M, Shan X, Gregory-Evans K. Gene-specific differential response to anti-apoptotic therapies in zebrafish models of ocular coloboma. Mol Vis 2011; 17: 1473-1484.

98 Bailey TJ, Davis DH, Vance JE, Hyde DR.

Spectral-domain optical coherence tomography as a noninvasive method to assess damaged and regenerating adult zebrafish retinas. Invest Ophthalmol Vis Sci 2012; 53(6): 3126-3138.
99 Meyers JR, Hu L, Moses A, Kaboli K, Papandrea A, Raymond PA. beta-catenin/Wnt signaling controls progenitor fate in the developing and regenerating zebrafish retina. Neural Dev 2012; 7: 30.

100 Ramachandran R, Zhao XF, Goldman D. Asclla/Dkk/betacatenin signaling pathway is necessary and glycogen synthase kinase-3beta inhibition is sufficient for zebrafish retina regeneration. Proc Natl Acad Sci USA 2011; 108(38): 15858-15863. 